\title{
A NEURAL CODE FOR AUDITORY SPACE IN THE CAT'S SUPERIOR COLLICULUS ${ }^{1}$
}

\author{
JOHN C. MIDDLEBROOKS ${ }^{2}$ AND ERIC I. KNUDSEN \\ Department of Neurobiology, Stanford University School of Medicine, Stanford, California 94305
}

Received February 16, 1984; Revised May 14, 1984; Accepted May 16, 1984

\begin{abstract}
We have examined the intermediate and deep layers of the cat's superior colliculus for evidence of a neural representation of auditory space. We measured the responses of single units to sounds presented in a free field. The results support the following generalizations. (1) Most auditory units in the superior colliculus have sharply delimited receptive fields which form two discrete classes distinguished by their locations and sizes. The remaining units respond to sounds presented at any location. (2) Each auditory unit responds maximally to sounds at a particular horizontal and vertical location within its receptive field, the unit's "best area." (3) The best areas and receptive field borders of a unit are resistant to changes in the intensity of stimulus. (4) The locations of best areas shift systematically as a function of unit position to form a continuous map of auditory space. The horizontal dimension of space is mapped rostrocaudally, and the vertical dimension is mapped mediolaterally. This map corresponds in orientation with the map of visual space.

These data permit us to infer the distribution of unit activity elicited by a sound at any given location. Regardless of its location, a sound activates a substantial portion of the superior colliculus. Indeed, sounds at some locations activate nearly all of the auditory units. However the activated portion of the colliculus contains a restricted region of units which are excited to near their maximum firing rates. The position of this focus of greatest activity varies systematically according to the location of the sound source, thus mapping the location of the sound in space.
\end{abstract}

How is the location of a sound source represented within the central auditory system? In the visual and somatosensory systems, primary afferents and central neurons are activated only by stimuli presented within delimited ranges of locations, or receptive fields, and a single stimulus presumably elicits activity within a small fraction of the total neuronal population. In contrast, fibers in each auditory nerve can respond to a sound of sufficient intensity regardless of its location. Hence, the location of a sound must be derived centrally from the acoustic cues, such as interaural differences in timing and intensity, that are provided by the passive acoustics of the head and external ears. Because of the different mechanisms involved, the characteristics of the central representation of auditory space cannot be predicted from what is understood about space coding in other sensory systems.

The superior colliculus is a structure that is primarily concerned with sensory and motor space: it mediates orienting movements of the head, eyes, and external ears to the sources of visual, somatic, and auditory stimuli (e.g., Gordon, 1973; Roucoux et al., 1981; Stein and Clamann, 1981; Wurtz and

\footnotetext{
${ }^{1}$ We are pleased to acknowledge the technical assistance of Phyllis F. Knudsen. This manuscript benefitted from critical readings by Drs. S. D. Esterly, M. Konishi, M. M. Merzenich, A. Moiseff, and C. J. Shatz. This research was supported by March of Dimes Foundation Grant 5-285 and National Institutes of Health Grants RO1 NS1609903 and 5F32 NS06992-02.

${ }^{2}$ To whom correspondence should be addressed, at Department of Neurobiology, Fairchild Science Building, Stanford University School of Medicine, Stanford, CA 94305.
}

Albano, 1981). Visual space and somatic space are represented topographically in the intermediate and deep layers (Gordon, 1973; Dräger and Hubel, 1976; Stein et al., 1976; Tiao and Blakemore, 1976; Chalupa and Rhoades, 1977). Similarly, auditory units in the superior colliculus are selective for the locations of sounds, and several reports have described maps of auditory space (Gordon, 1973; Harris et al., 1980; Knudsen, 1982; King and Palmer, 1983).

Previous studies have used several different measures to characterize the location selectivity of auditory units (Gordon, 1973; Dräger and Hubel, 1975; Chalupa and Rhoades, 1977; Harris et al., 1980; Knudsen, 1982; King and Palmer, 1983). Most investigators have used unit receptive fields, the regions within which sounds elicit excitatory responses (e.g., Gordon, 1973; Knudsen, 1982). The locations of receptive fields have been represented by some groups by their geometrical centers (Ilarris et al., 1980), but other workers have found auditory receptive fields to be large and poorly delimited and have regarded the most frontal border, or "leading edge," as the most salient feature of location selectivity (Gordon, 1973). Other groups have plotted spatial response profiles by measuring the responses of units to sounds varied systematically in location; these groups have focused on the peak of the spatial response profile, or "best area," of units in describing spatial tuning (Knudsen, 1982; King and Palmer, 1983).

We have examined several features of the location selectivity of single units in the cat's superior colliculus with the aim of identifying those features which vary as a function of unit position to form a map of auditory space. We compared the receptive field centers, receptive field leading edges, and best 
areas of units as well as the manifestations of these parameters in the spatial pattern of activity across the population of auditory units. Each of these features varies systematically throughout the colliculus, yet only by measuring the best areas of units could we demonstrate a continuous and complete map of the horizontal and vertical dimensions of auditory space.

\section{Materials and Methods}

We recorded the responses of units to auditory stimuli presented in a free sound field. For some units, visual receptive fields also were measured. Auditory responses were recorded from 178 single units and 106 small clusters of units located in the intermediate and deep layers of the superior colliculi of 29 cats. Data collected from the unit clusters were consistent with the single unit data, but only the single unit data are illustrated.

Animal preparation. Adult cats were selected for clean ears. Anesthesia was induced with an intramuscular injection of ketamine hydrochloride $(30 \mathrm{mg} / \mathrm{kg})$. Diazepam $(1 \mathrm{mg} / \mathrm{kg})$ or acepromazine maleate $(0.25 \mathrm{mg} / \mathrm{kg})$ was given intramuscularly to eliminate the muscular hypertonia resulting from the ketamine (Green et al., 1981), and atropine $(50 \mu \mathrm{g}, \mathrm{i} . \mathrm{m}$.) was given to suppress salivation. Cats were maintained at an areflexic level of anesthesia during all surgical procedures. During recording sessions, we used supplementary injections of ketamine, alone or occasionally with additional diazepam or acepromazine, to maintain the cat at a level of anesthesia at which corneal reflexes could be elicited but no spontaneous movements occurred. This anesthetic protocol was chosen because it produced adequate analgesia and restraint without blocking activity in the deeper layers of the superior colliculus; the deeper layers are reported to be relatively unresponsive under barbiturate anesthesia (Sterling and Wickelgren, 1969). A tracheostomy was performed. The scalp was opened, and a small metal plate containing threaded sockets was fastened to the skull rostral to the coronal suture (lambda) using stainless steel screws and dental acrylic. A skull opening was made $1 \mathrm{~cm}$ caudal to the coronal suture, and the dura was opened. We infiltrated the wound margins with long-lasting local anesthetic (Marcaine) and inserted contact lenses into the eyes to protect the corneas from drying.

The cat was positioned in a darkened sound-attenuating chamber that was carpeted and fitted with Fiberglas panels to suppress sound reflections. The cat's body was held in a canvas sling and its head was held from behind by a steel bar attached to the metal skull plate. The head was oriented with the Horsley-Clarke horizontal plane of the cat tilted back about $30^{\circ}$ from the plane of the floor. The variability in head orientation between cats was estimated in several cases post mortem by making pairs of cuts in the brain, one in the plane of the electrode penetrations and one in the Horsley-Clarke coronal plane. In six cats, the orientation of the Horsley-Clarke horizontal plane ranged from 26 to $33^{\circ}$ (median $29^{\circ}$ ).

We observed that a cat that is stalking a sound source holds its external ears in a symmetrical position with the medial margins lying in a frontal plane. In the anesthetized cats, we held the ears in this position using wound clips and sutures attached to the scalp. The positions of the head and external ears were constant throughout each experiment. We measured the positions of the eyes with an ophthalmoscope by plotting the locations of the optic disks. Under anesthesia, the eyes tended to remain constant in position within a few degrees. The mean angle of the optic disks in 42 measurements in 13 cats was $35^{\circ}$ above the floor, which indicates that the visual fixation plane was near the Horsley-Clarke horizontal plane (Bishop et al., 1962).

Auditory and visual stimulation. Auditory stimuli were presented through a small loudspeaker that could be positioned by remote control at any location on the surface of an imaginary sphere, $92 \mathrm{~cm}$ in radius, centered on the cat's head (Knudsen, 1982). The loudspeaker was stationary during all stimulus presentations. We calibrated the sound source with a Bruel and Kjaer sound level meter, using the " $\mathrm{A}$ " weighting scale for noise and one-third octave filters for tones. Sound pressure levels ( $\mathrm{dB}$ SPL) are expressed relative to $20 \mu \mathrm{Pa}$. The frequency response of the loudspeaker was constant within $\pm 2 \mathrm{~dB}$ in the range of 1.9 to $12 \mathrm{kHz}$ and was attenuated by $12 \mathrm{~dB}$ at 1.4 and $40 \mathrm{kHz}$. Noisc bursts werc 50 msec in duration, gated with abrupt onsets and offsets, and were presented once every 2 sec. Tone bursts were shaped with $5-\mathrm{msec}$ rise and fall times. The characteristic frequency of a unit is defined as the tonal frequency to which it is most sensitive. The sharpness of frequency tuning is expressed by the $Q_{10 \mathrm{~dB}}$ which is the characteristic frequency divided by the width of the band of frequencies to which the unit responds at $10 \mathrm{~dB}$ above threshold. The frequency tuning and threshold to noise stimulation for a given unit were measured with the loudspeaker positioned within the unit's best area.

Visual stimuli were projected with a hand-held projector onto a translucent hemisphere (radius $57 \mathrm{~cm}$ ); the hemisphere was positioned in front of the cat for visual testing and was removed during auditory testing. Stimuli consisted of moving spots and bars of light. For units that had well delimited visual receptive fields, the location of a receptive field was specified by its geometric center. For units with poorly defined field boundaries, the area in which stimuli elicited the strongest response was specified as the "center." Visual data were discarded in the few cases in which the eycs appeared to be moving or when the optic disks were displaced by more than $5^{\circ}$ from their resting position. Visual field locations are shown without adjustment for eye positions.

We indicated the locations of auditory and visual stimuli by the angles formed at the center of the cat's head by the sound source and two reference planes. The horizontal plane of the coordinate system coincided within a few degrees with the Horsley-Clarke horizontal plane and the visual fixation plane of the cat (see "Animal preparation," above). The median plane coincided with the cat's midsagittal plane. A third plane, the interaural transverse plane, was the plane that was orthogonal to the horizontal plane and contained the interaural axis; the interaural axis is the line passing through the two bony acoustic meati. The horizontal location of a stimulus was indicated by its azimuth coordinate. This was the angle formed with the median plane and was ipsi- or contralateral with respect to the recording site. Azimuths in front of the interaural transverse plane range from 0 to $90^{\circ}$ ipsi- or contralateral, and azimuths behind the interaural transverse plane range from 90 to $180^{\circ}$ ipsi- or contralateral. The vertical location of a stimulus was indicated by its elevation coordinate. Elevation was the angle above $(+)$ or below $(-)$ the horizontal plane. This is a double pole coordinate system (Knudsen, 1982), which has the advantage that azimuth and elevation coordinates are mutually independent. In this system, a given angle in elevation corresponds to a constant length on the unit sphere regardless of the azimuth at which it is measured.

Unit recording. Extracellular unit activity was recorded with glassinsulated tungsten microelectrodes. Electrode penetrations were directed ventrally and slightly caudally, about $30^{\circ}$ from the HorsleyClarke coronal plane. The brain tissue lying over the superior colliculus was left intact. The locations of penetrations were referred to the midline and to the coronal suture. All recordings were made from the right superior colliculus. Visual stimuli were used as search stimuli to locate the superior colliculus, then noise bursts were used to locate auditory units in the deeper layers. Unit activity was displayed on an oscilloscope and an audio monitor. Spikes were discriminated with a level discriminator, plotted as a raster on a second oscilloscope, and counted with an event counter.

We characterized the location selectivity of units by measuring the boundaries of their excitatory receptive fields and by compiling spatial response profiles. Spatial respose profiles were measured by varying the location of the loudspeaker systematically in azimuth and/or elevation and counting the number of spikes elicited by eight noise bursts presented at each stimulus location. Ranges of azimuth or elevation were sampled by shifting the loudspeaker in equal steps in one direction, then reversing direction to sample the intervening locations. Data were collected in angular increments of 5 to $20^{\circ}$. When units appeared to fire erratically, we measured responses to two or three sets of eight noise bursts presented at each speaker location and used the median response at each location to draw the profile. The spatial response profile and the receptive field borders of a unit were measured in two separate procedures; thus spatial profiles were not necessarily measured to the edges of the receptive field.

Histological procedures. The locations of recording sites were marked with electrolytic lesions (Fig. 1). After recording sessions, cats were deeply anesthetized with sodium pentobarbital and perfused through the heart with $0.1 \mathrm{M}$ phosphate buffer, $\mathrm{pH} 7.4$, containing $4 \%$ sucrose followed by buffered $4 \%$ formaldehyde. The brains were blocked parasagittally or transversely in the plane of the electrode penetrations, soaked in buffered $30 \%$ sucrose, and then sectioned frozen at $40 \mu \mathrm{m}$. Alternate sections were stained with cresyl violet for Nissl substance and with a hematoxylin stain for myelin (Morgan, 1926). We used a camera lucida to mark the locations of lesions on drawings of the sections.

The layers of the superior colliculus were identified according to the system of Sprague and Miekle (1965) and Kanaseki and Sprague (1974). 

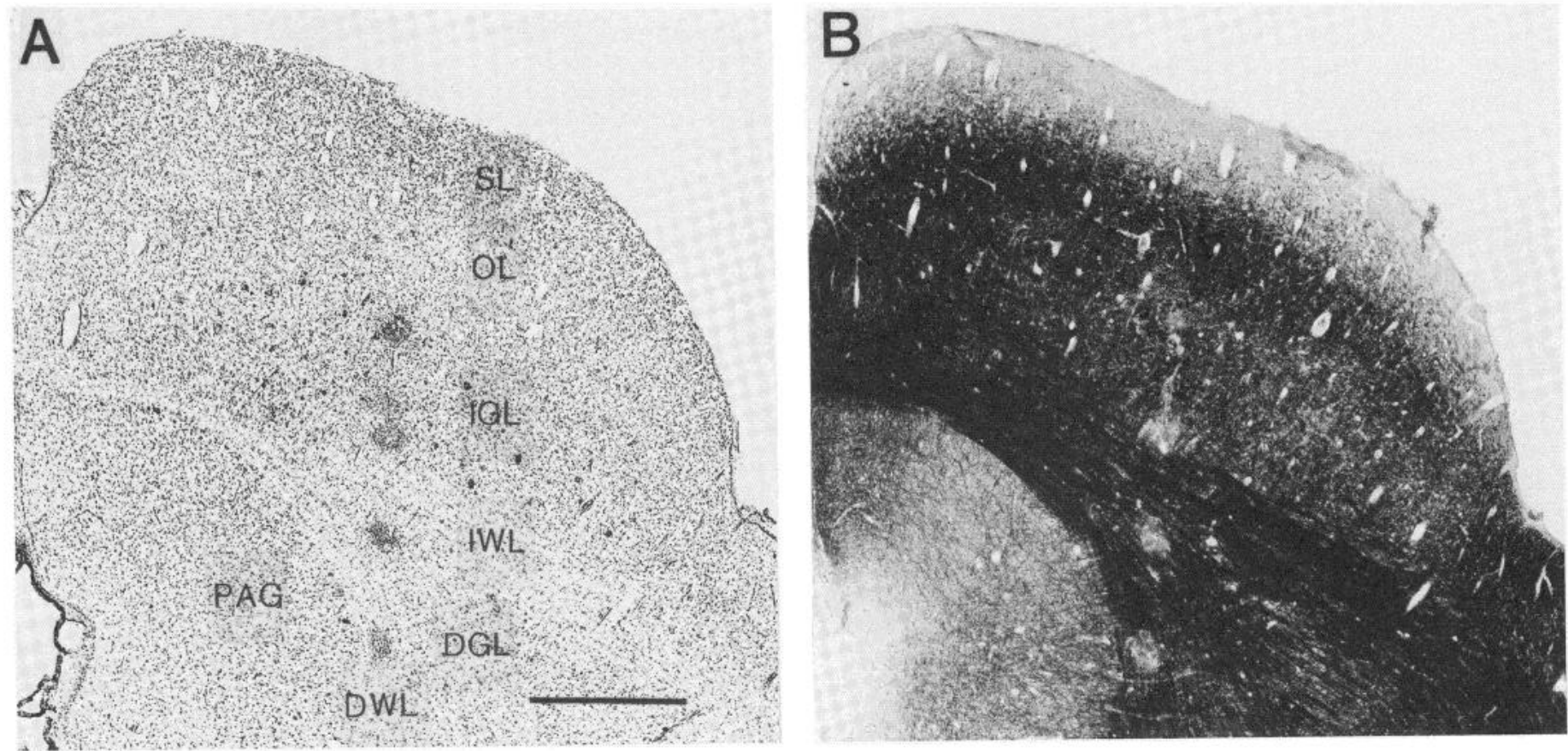

Figure 1. Layers of the superior colliculus. These adjacent sections were cut in a transverse plane parallel to the plane of the electrode penetrations, approximately $30^{\circ}$ from the Horsley-Clarke coronal plane. Four electrolytic lesions along a single penetration are visible in each section. The sections were stained with cresyl violet $(A)$ and hematoxylin $(B)$. $D G L$, deep gray layer; $D W L$, deep white layer; $I G L$, intermediate gray layer; $I W L$, intermediate white layer; $O L$, optic layer; $P A G$, periaqueductal gray; $S L$, superficial layers. $S c a l e: 1 \mathrm{~mm}$.

The intermediate gray layer (stratum griseum intermedium) contained medium to large cells and was relatively free of myelin. A distinct band of myelinated fibers marked the intermediate white layer (stratum album intermedium). The deep gray layer (stratum griseum profundum) contained medium to large cells and less myelin than the intermediate white layer.

\section{Results}

General features of auditory and visual responses. Layers of the superior colliculus showed characteristic patterns of spontaneous and stimulus-evoked activity. In the superficial layers, visual stimuli elicited responses in many small units; the multiunit receptive fields were less than $10^{\circ}$ in diameter. In the optic layer, visual responses were more robust and the multiunit visual receptive fields often were larger. Deep in the optic layer, we usually recorded a graded auditory evoked potential.

The most superficial auditory units were located in the intermediate gray layer. Most of these units also responded to visual stimulation and had visual receptive fields substantially larger than those found in more superficial layers. In the intermediate white layer, auditory stimuli elicited robust multiunit activity and a multiphasic graded potential; it was difficult to isolate single units in this layer. Visual responses seldom were found. In the deep gray layer, auditory responses were not distinguishable from those in the intermediate gray layer. The deep gray layer differed in that no visual responses were recorded.

We recorded auditory responses in every electrode penetration that passed through the intermediate and/or deep layers of the superior colliculus. Single units isolated in those layers commonly showed little or no spontaneous activity. In response to noise presented from optimal locations, most auditory units responded with a single spike locked to the onset of the stimulus followed by one or more additional spikes. Some units responded continuously throughout the duration of a noise burst. Latencies were measured relative to the estimated time of arrival of the stimulus at the tympanum. The median latency to abrupt onset noise bursts was $10 \mathrm{msec}$ (range, 5 to $29 \mathrm{msec}$;
$N=110$ ). The latencies among all of the auditory layers were essentially equal, although the sample from the deepest layers was too small to permit a detailed comparison.

We often observed changes in the excitability of units. Many units habituated to stimuli presented as often as once per second. Short-term fluctuations in excitability appeared as variability in the number of spikes elicited by individual noise bursts within a series of eight trials. In the long term, the general responsiveness of a unit sometimes decreased or increased over the course of many stimulus presentations. Problems introduced by changing excitability were minimized by repeating measurements at some speaker locations and sometimes by randomizing the order in which different portions of a unit's receptive field were sampled.

Unit thresholds to sound in the superior colliculus were somewhat higher than those normally measured in the primary auditory pathway. Noise thresholds typically fell in the range of 0 to $20 \mathrm{~dB}$ SPL. This range was approximately $10 \mathrm{~dB}$ higher than the thresholds that we have measured in the inferior colliculus (unpublished observations). Most auditory units in the superior colliculus responded poorly to tonal stimuli. Of the 40 units that were tested systematically with tones, only 18 (45\%) had tonal thresholds less than $40 \mathrm{~dB}$ SPL. Most units that responded to tones were broadly tuned for frequency. Only 9 of the 40 units tested had a $Q_{10 \mathrm{~dB}}$ greater than 1.0. Of the 9 sharply tuned units, 7 had characteristic frequencies of $19 \mathrm{kHz}$ or greater, and 8 had best areas centered within $35^{\circ}$ of the frontal midline. Tonal thresholds generally were higher than the noise thresholds even for the most sharply tuned units. This is despite the fact that a noise at any particular sound pressure level contains substantially less energy at a unit's characteristic frequency than a characteristic frequency tone of the same sound pressure level.

Auditory spatial selectivity. All units recorded in the superior colliculus were selective for sound location. Most units had sharp receptive field borders, and most showed a prominent best area. No consistent difference in spatial selectivity was observed between the different auditory layers. 
A unit's receptive field was defined as the region within which noise bursts activated the unit above its resting firing level. Most units had no resting discharge; therefore, for these units a single stimulus-locked spike in eight stimulus epochs was considered to be an excitatory response. The response of a typical unit would decline from at least 2 spikes $/ 8$ stimulus epochs to 0 spikes as the sound source was moved through an angle of about $10^{\circ}$ crossing a receptive field border. Receptive field borders were measured to the nearest 5 or $10^{\circ}$. Three classes of units were distinguished by their receptive field properties. The receptive fields of frontal units were contained entirely in front of the interaural transverse plane; these units accounted for $38 \%$ of the 90 single units for which receptive fields were measured. The receptive fields of hemifield units extended behind the cat's head; these units accounted for $54 \%$ of the population. Figure 2 shows examples of the receptive fields of frontal and hemifield units. The remaining $8 \%$ of the units responded to sounds presented at all tested locations; these units were named omnidirectional.

The receptive fields of frontal and hemifield units differed in their locations and sizes (Fig. 3). The geometrical centers of receptive fields were distributed discontinuously in azimuth, with no fields centered between contralateral 40 and $70^{\circ}$. All of the fields of hemifield units were centered within $20^{\circ}$ of the interaural axis (i.e., between contralateral 70 and $110^{\circ}$ ), and all of the fields of frontal units were centered between $0^{\circ}$ and contralateral $40^{\circ}$ which is the approximate region of greatest sensitivity of the contralateral external ear (Middlebrooks and Pettigrew, 1981; Phillips et al., 1982). The sizes of receptive fields, indicated by their widths in azimuth, also were strongly bimodal. Most of the frontal receptive fields were $90^{\circ}$ or smaller in width and most of the hemifield receptive fields were larger than $110^{\circ}$. Note that the population of hemifield units is somewhat underrepresented in the illustrations since the pos- terior borders of these receptive fields were not always measured.

Units with different classes of receptive fields were segregated within the superior colliculus. Figure 4 shows the positions of hemifield, frontal, and omnidirectional units in a dorsal view of the intermediate gray layer. Frontal units and omnidirectional units were located rostral to hemifield units.

Units were selective for sound locations within their receptive fields. Spatial response profiles in azimuth and elevation are

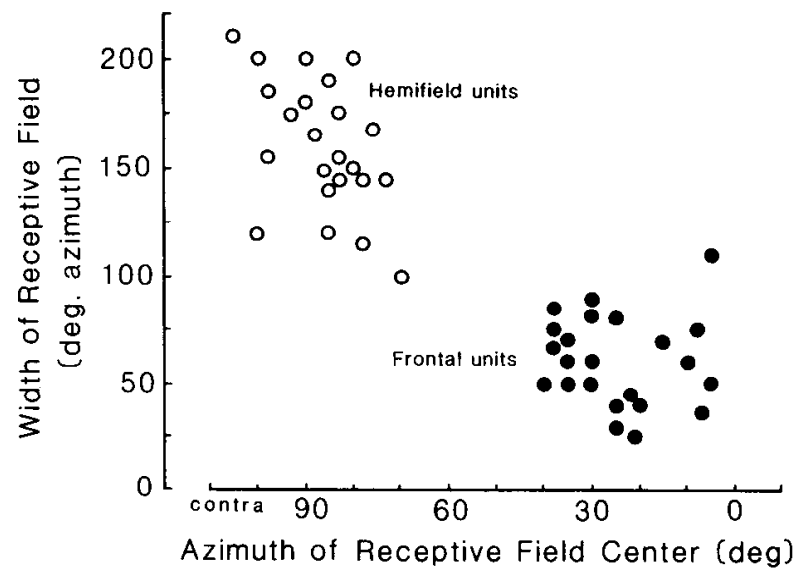

Figure 3. Widths of receptive fields as a function of their locations. The location of a receptive field is given by the location in azimuth of its gcometric center, and the width is its greatest extent in azimuth. Open circles represent hemifield units, and solid circles represent frontal units. In this and subsequent figures, data shown for each unit are for the greatest sound level at which the unit was tested. This always was at least $10 \mathrm{~dB}$ above the threshold of each unit measured at its best area. Only fields that were measured with a precision of $\pm 5^{\circ}$ are shown.

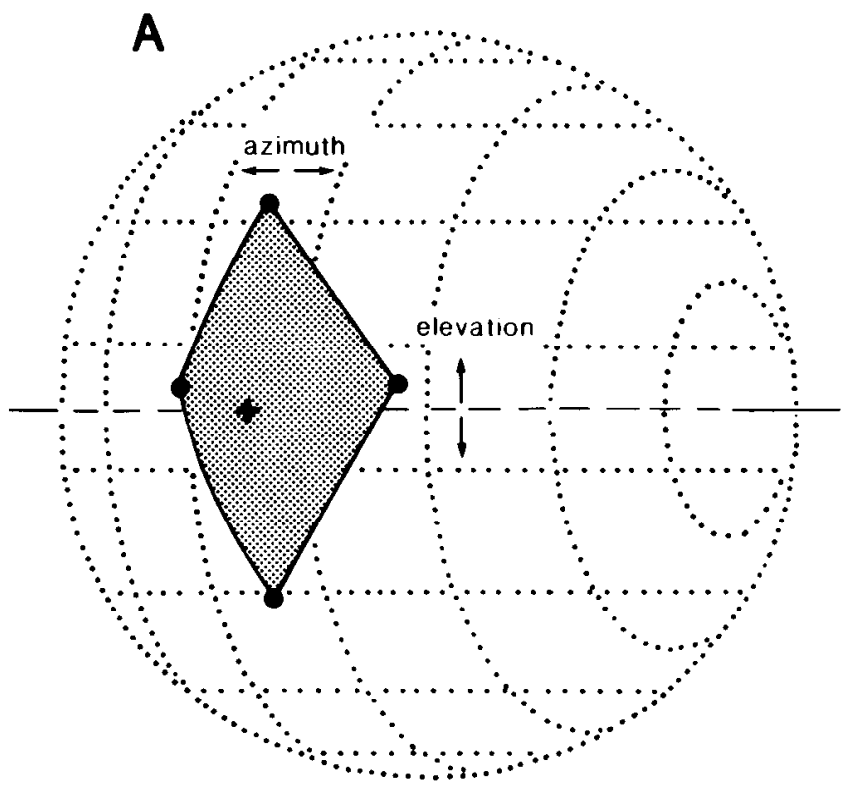

Frontal

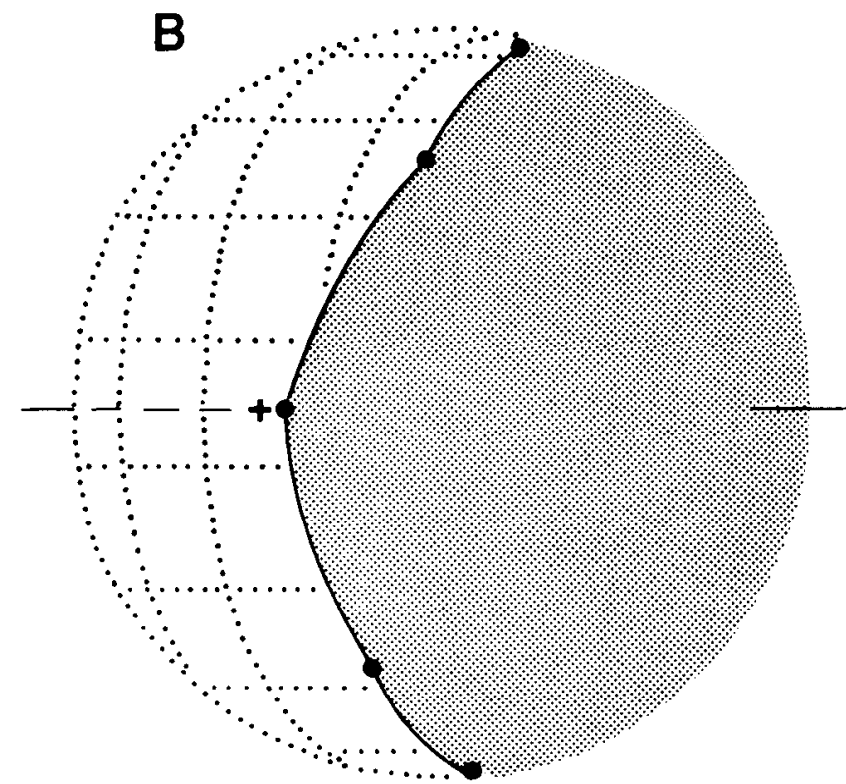

Hemifield

Figure 2. Receptive fields of frontal and hemifield units. The shading indicates areas within which stimuli elicited responses greater than the spontaneous firing rate of each unit. The solid circles indicate the locations at which receptive field borders were measured. The crosses indicate the intersection of the median plane with the visual horizontal plane, i.e., $0^{\circ}$ azimuth, $0^{\circ}$ elevation. The straight lines passing through the coordinate globes represent the interaural axis. Fields in $A$ and $B$ were measured using stimulus intensities of 50 and $40 \mathrm{~dB}$ SPL, respectively, which were $30 \mathrm{~dB}$ above the threshold of each unit at its best area. $A$, Frontal units had receptive fields that were contained entirely in front of the interaural transverse plane. $B$, Hemifield units had receptive fields that extended behind the cat's head. The fields of hemifield units were approximately symmetrical with respect to the interaural axis. 
shown in Figure 5 for a frontal unit and in Figure 6 for a hemifield unit. Each spatial profile shows a single area of optimal stimulus locations surrounded by locations from which stimuli elicited responses that were above the unit's resting firing level yet well below its maximum response. Spatial response profiles revealed spatial selectivity even when receptive fields were unbounded, such as for all omnidirectional units

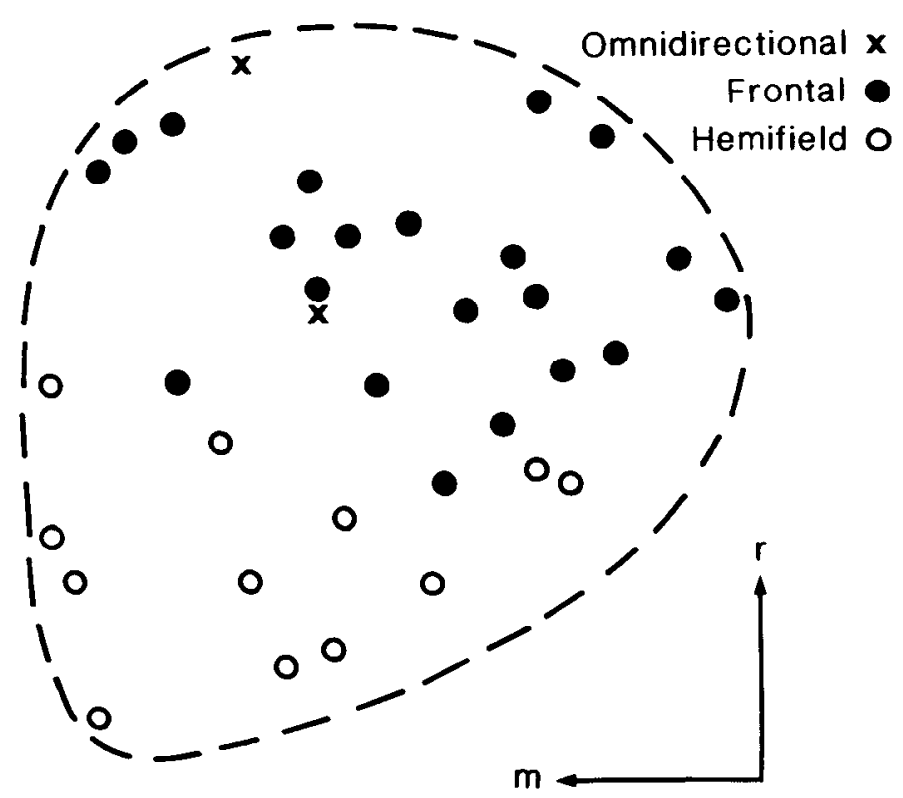

Figure 4. Distribution of unit classes within the intermediate gray layer. This dorsal view of the intermediate gray layer illustrates the locations of hemifield $(O)$, frontal $(\bullet)$, and omnidirectional unils $(X)$. The approximate extent of the layer is indicated with a dashed line. These 35 units were recorded in 35 electrode penetrations in seven cats. $r$, rostral; $m$, medial. Scale $=1 \mathrm{~mm}$. and for many hemifield units in elevation (e.g., Fig. 6C). In some recordings (not illustrated), single units could not be isolated, and we studied the location selectivity of clusters of several units. The response profiles of these clusters often were as sharp as those of single units, indicating that the spatial tuning of single units was representative of tuning of populations of neurons located near each recording site.

To provide an objective indicator of the optimal stimulus location for each unit, we defined the best area of a unit as the region within which a given stimulus elicited a response greater than $75 \%$ of the unit's maximum response to that stimulus. The $75 \%$ criterion was chosen because it fell near the steepest part of the spatial response profile of most units; this response level is indicated in Figures 5 and 6 by dashed arcs. Occasionally, a few measurements in a unit's spatial response profile would fluctuate around the $75 \%$ level; in such cases, the best area was taken to be the maximum area in which the above criterion responses were recorded.

The location of a unit's best area relative to its receptive field borders varied among units. In Figure 7, the locations of best areas and receptive fields are represented by the locations in azimuth of their geometrical centers. Best areas and receptive fields were approximately concentric for most frontal units. In contrast, the centers of best areas of hemifield units were located as far frontal as contralateral $35^{\circ}$, even though their receptive fields all were centered peripheral to contralateral $70^{\circ}$. For example, the unit shown in Figure 6 had its best area centered $40^{\circ}$ frontal to the center of its receptive field. Units for which receptive field centers were undefined, i.e., the omnidirectional units, had best areas located near the frontal midline.

Units varied in the sharpness of their spatial selectivity; the variation measured within a single cat was as great as that measured among different cats. We used the size of a unit's best area as an indicator of the sharpness of its spatial tuning. The widths of best areas and the amount of variation in their widths were greatest for the most peripheral best areas (Fig.
A

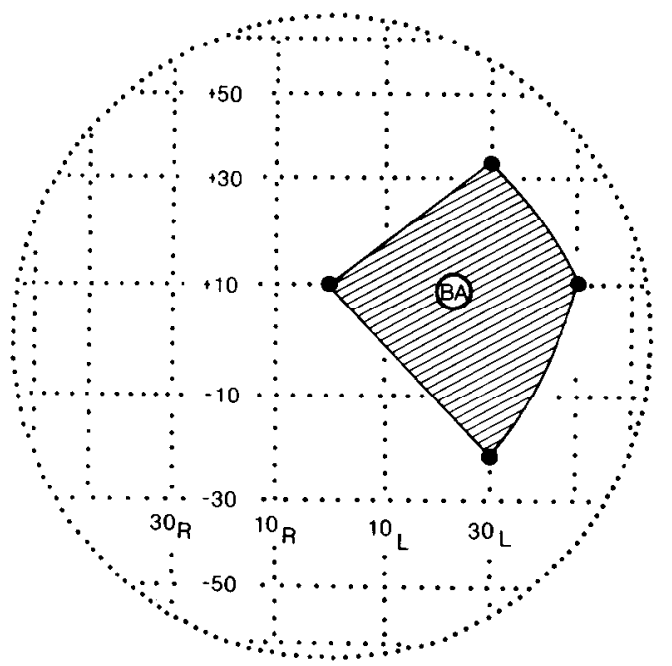

B

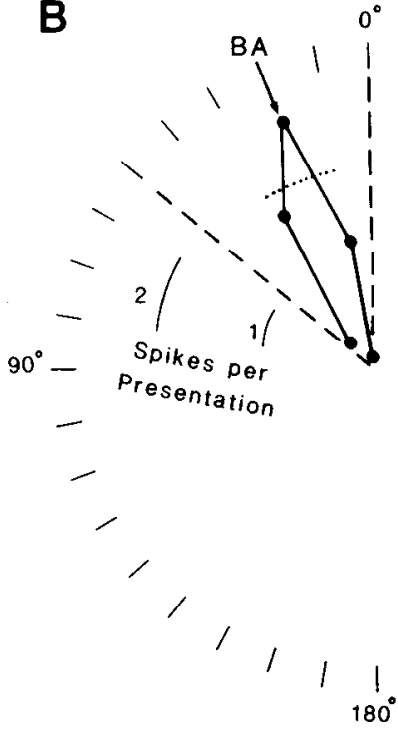

C

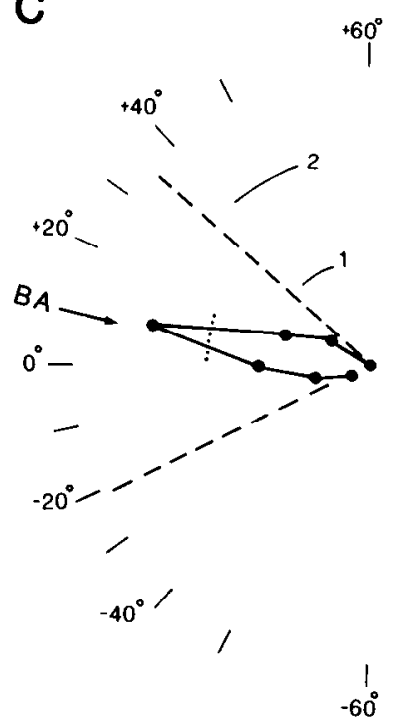

Figure 5. Spatial tuning of a frontal unit. The globe in $A$ shows the location of the unit's receptive field (hatching) and the center of its best area (clear area labeled $B A$ ). The polar plots represent the responses of the unit to sounds presented at successive locations in azimuth $(B)$ and elevation $(C)$. The angular dimension indicates the stimulus location and the radial dimension indicates the mean response rate (total number of spikes divided by the number of noise bursts). The dashed lines in $B$ and $C$ indicate the borders of the receptive field in azimuth and elevation. The dashed arcs indicate the $75 \%$ response criterion. The best area is the area within which stimuli elicited responses greater than this criterion. The arrows indicate the center of the best area in azimuth and elevation. The stimulus intensity was $40 \mathrm{~dB}$ SPL, $32 \mathrm{~dB}$ above the threshold of the unit at its best area. The elevation profile in $C$ was measured at $30^{\circ}$ azimuth. The most extreme elevations possible at this azimuth are $60^{\circ}$ above (+) and below (-) the horizontal plane as indicated by the labels in $C$. 

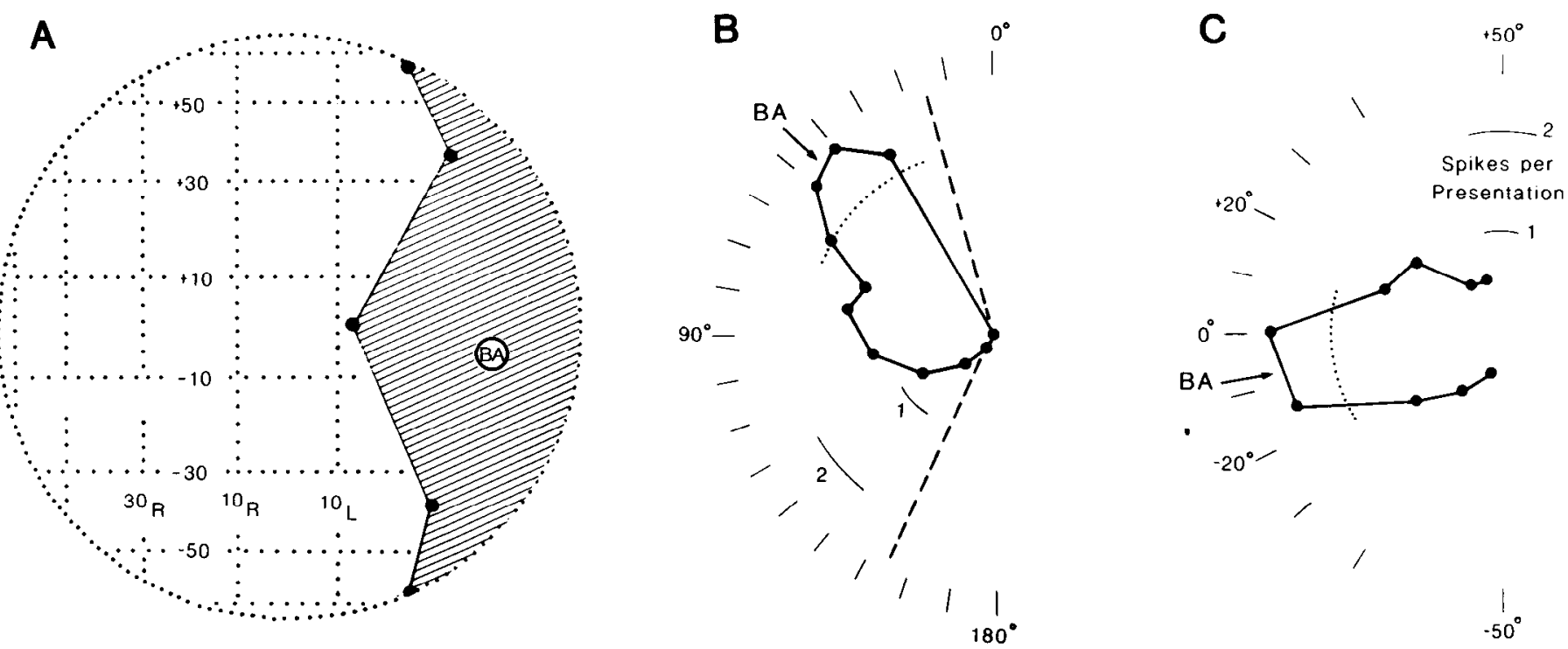

Figure 6. Spatial tuning of a hemifield unit. The stimulus intensity was $29 \mathrm{~dB}$ SPL, $15 \mathrm{~dB}$ above the threshold of the unit at its best area. The elevation profile in $C$ was measured at $40^{\circ}$ contralateral azimuth. Although the spatial response profile of this unit demonstrates clear seleclivity in elevation, the unit responded above its resting levels to sounds at all elevations at this azimuth. Other details are as in Figure 5.

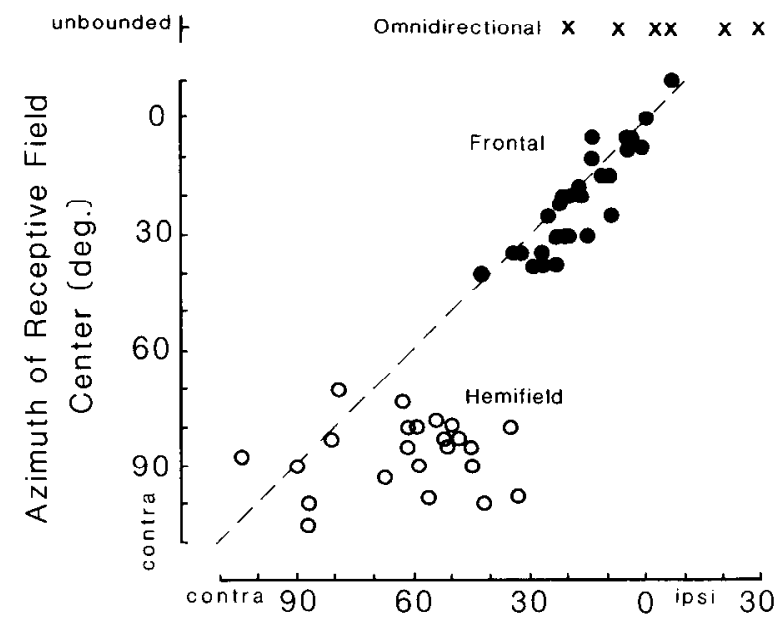

Azimuth of Best Area Center (deg.)

Figure 7. Locations of receptive fields versus locations of best areas. Data are from hemifield $(O)$, frontal $(\bullet)$, and omnidirectional $(X)$ units. The dashed line indicates perfect correspondence. Omnidirectional units had no receptive field boundaries, therefore, the location on the ordinate for these units is arbitrary. However, all omnidirectional units had best areas.

$8 A)$. Most of the largest best areas were centered in elevation above the horizontal plane (Fig. $8 B$ ). All of the units with best areas greater than $40^{\circ}$ in height were located at the medial edge of the superior colliculus. The best areas of most units were centered in the frontal contralateral quadrant between about $+10^{\circ}$ and $-30^{\circ}$ elevation.

The spatial tuning of most units was insensitive to changes in stimulus intensity for stimuli greater than approximately 10 $\mathrm{dB}$ above threshold. Figure 9 shows for one unit the receptive fields and spatial response profiles measured at intensities 15 and $30 \mathrm{~dB}$ above its threshold. An increase of $15 \mathrm{~dB}$ in the sound pressure level caused minimal changes in the receptive field, the response profile, or the maximum firing level. For a stimulus at a given location, the firing rates of most units increased sharply as the intensity was increased through the first $10 \mathrm{~dB}$ above threshold, then remained relatively constant during subsequent increases in intensity. Figure 10 shows three features of azimuth tuning plotted against intensity above threshold. Each line or pair of lines connects data points collected from one unit at two or more intensities. The locations of the centers of best areas and receptive fields showed no systematic change with increasing intensity. The leading edges (the most frontal borders) of most receptive fields shifted slightly ipsilaterally in response to increasing intensity, indicating that receptive fields tended to grow slightly in size as stimulus intensities were increased.

Auditory-visual alignment. Most auditory units in the intermediate gray layer also responded to visual stimulation. The centers of visual receptive fields coincided in general with the centers of auditory best areas. This correspondence is shown in Figure 11, $A$ and $B$. Small movements of the cat's eyes could account for much of the scatter that is evident, although eye movements probably could not account for the general tendency of auditory best area centers to lie somewhat peripheral to visual field centers (Fig. 11 $A$ ).

In contrast to best areas, auditory receptive fields did not correspond systematically with visual receptive fields (Fig. 11, $C$ and $D$ ). We observed a general correspondence in azimuth between the auditory and visual fields of frontal units. However, the auditory receptive fields of hemifield units were related to visual field locations only to the extent that the auditory fields always were centered peripheral to the centers of the visual fields. The omnidirectional units had visual fields located near the frontal midline but, of course, these unbounded units had no auditory receptive field centers. Similarly, auditory and visual receptive field centers seldom coincided in elevation (Fig. $11 D)$. Many of the auditory fields of hemifield units were symmetrical about the interaural axis, so that they were centered near elevation $0^{\circ}$, and others were unbounded in elevation. Yet, the visual fields of these units were distributed across a wide range of elevations.

Topography of auditory spatial tuning. In a series of mapping experiments, we related the spatial tuning of units to their positions in the superior colliculus. Data from 46 single units located in the intermediate gray layer were recorded in 46 electrode penetrations in seven cats. At least four of these units were recorded in each cat. Data from this population of units appear in Figures 12 to 17.

Best areas of single units varied systematically with unit position in the superior colliculus. Figure $12 \mathrm{~A}$ shows the azi- 

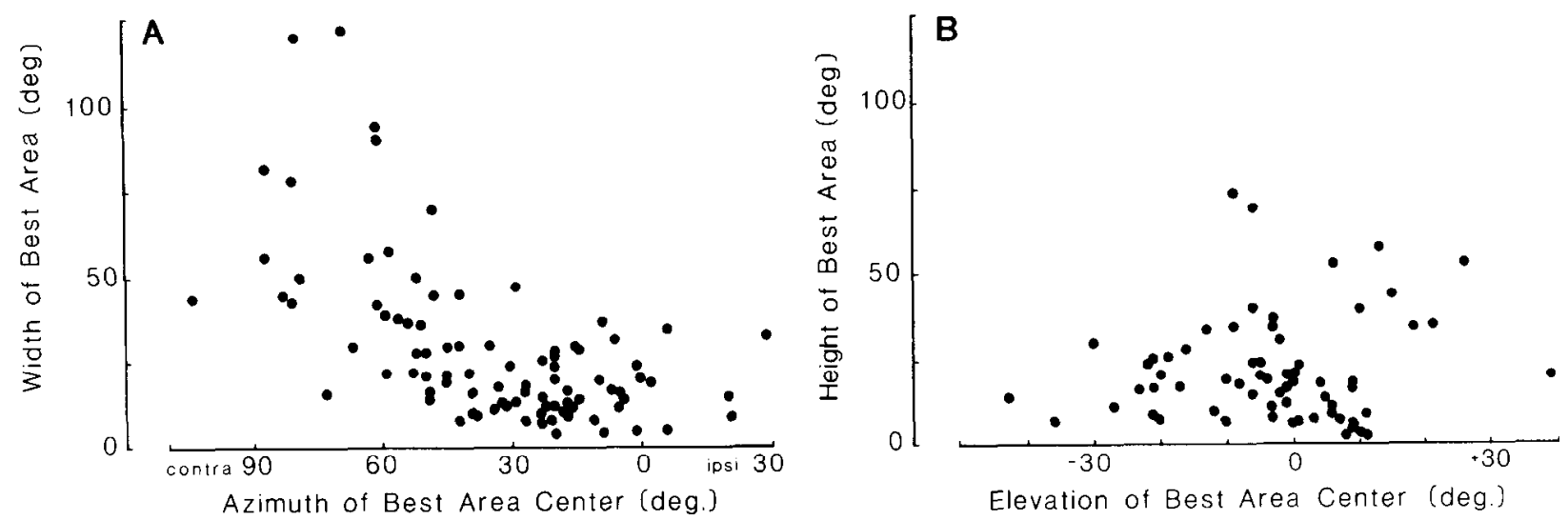

Figure 8 . Sizes of best areas versus locations of their geometrical centers. The widths $(A)$ and heights $(B)$ of best areas are plotted against their locations in azimuth and elevation, respectively.
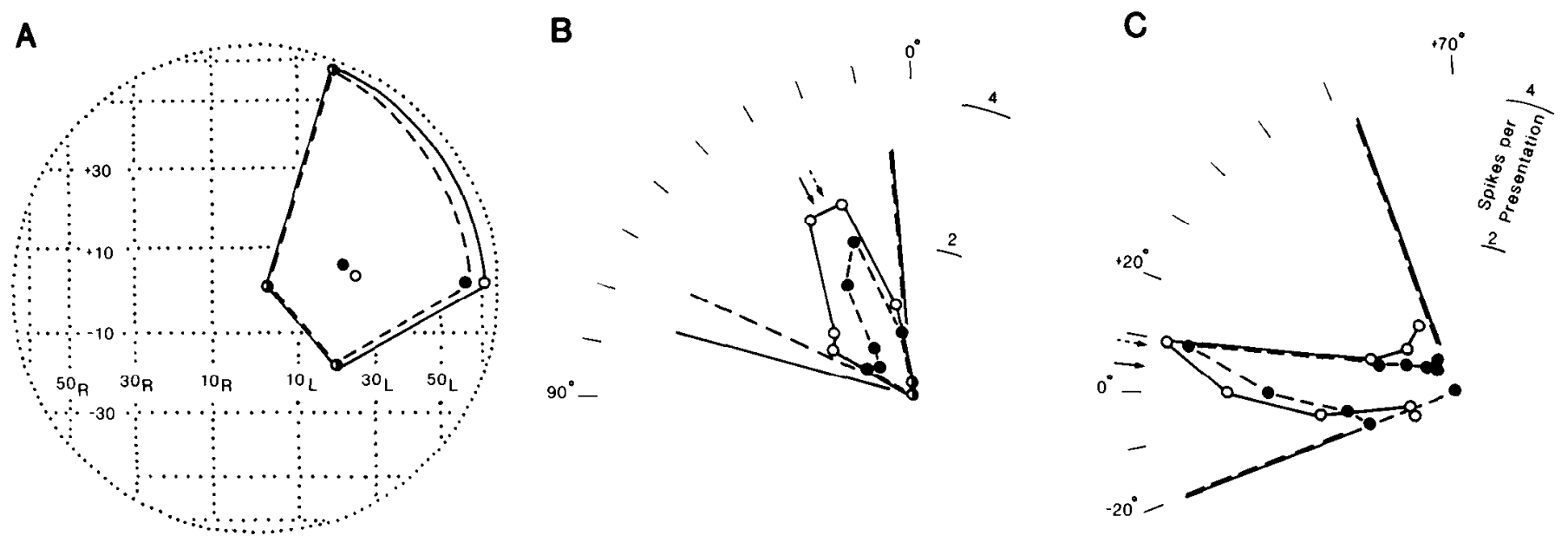

Figure 9. Effects of changing sound intensity on spatial tuning of a single unit. Dashed lines and solid circles indicate responses to a stimulus intensity of $30 \mathrm{~dB}$ SPL, and solid lines and open circles indicate measurements at $45 \mathrm{~dB}$ SPL. These levels were 15 and $30 \mathrm{~dB}$ above the threshold of the unit at its best area. The receptive fields measured at two intensities are shown in $A$. For this unit, only the lateral border of the receptive field shifted when the stimulus intensity was increased. The open and solid circles within the field indicate the centers of the best areas measured at the two intensities. $B$ and $C$ show the spatial tuning in azimuth and elevation, respectively. The elevation profile in $C$ was measured at contralateral $20^{\circ}$ azimuth. Other details are as in Figure 5.

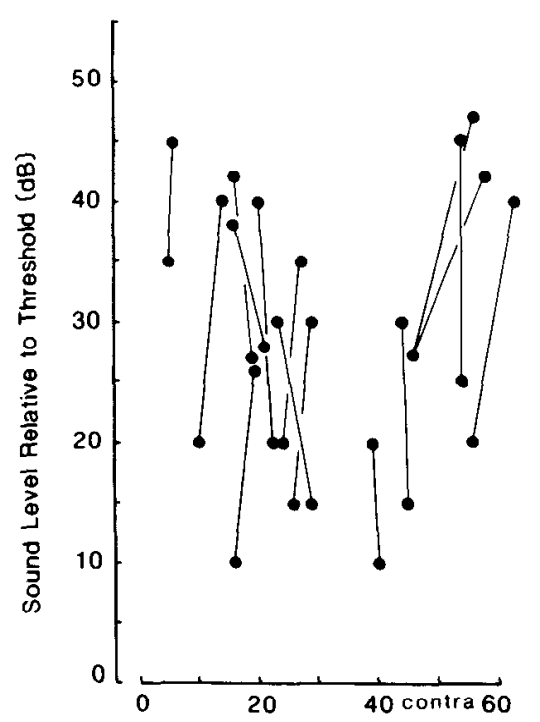

Azmuth of Best Area Center (deg)
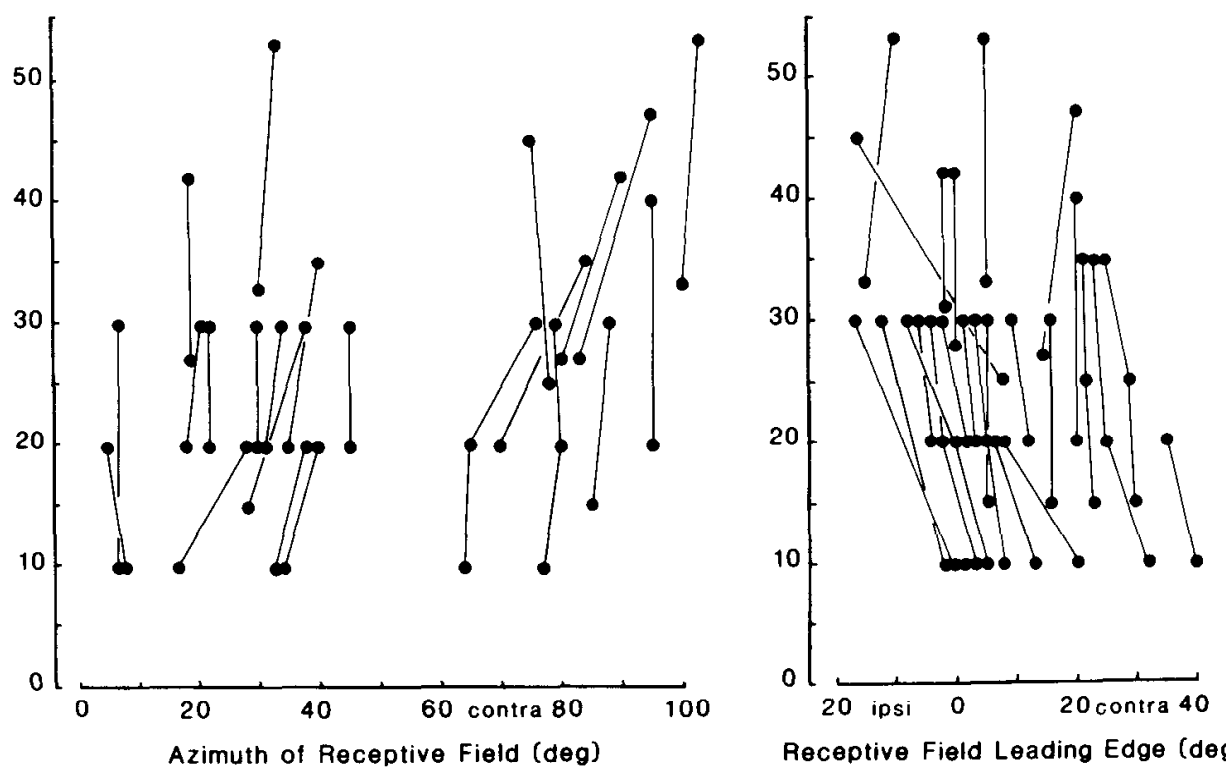

Receptive Field Leading Edge (deg)

Figure 10. The effect of changing stimulus intensity on the locations in azimuth of best area centers $(A)$, receptive field centers $(B)$, and receptive field leading edges $(C)$. Lines connect the data collected from each unit. Sound levels are expressed relative to the threshold of each unit measured within its best area. 

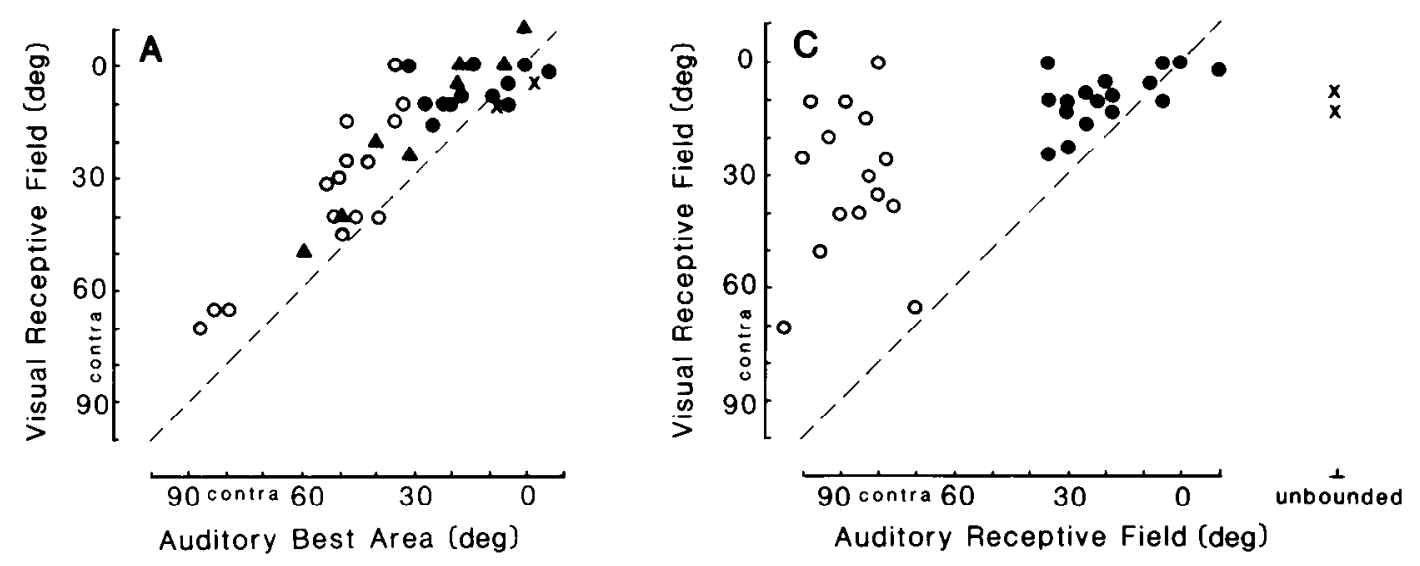

AZIMUTH
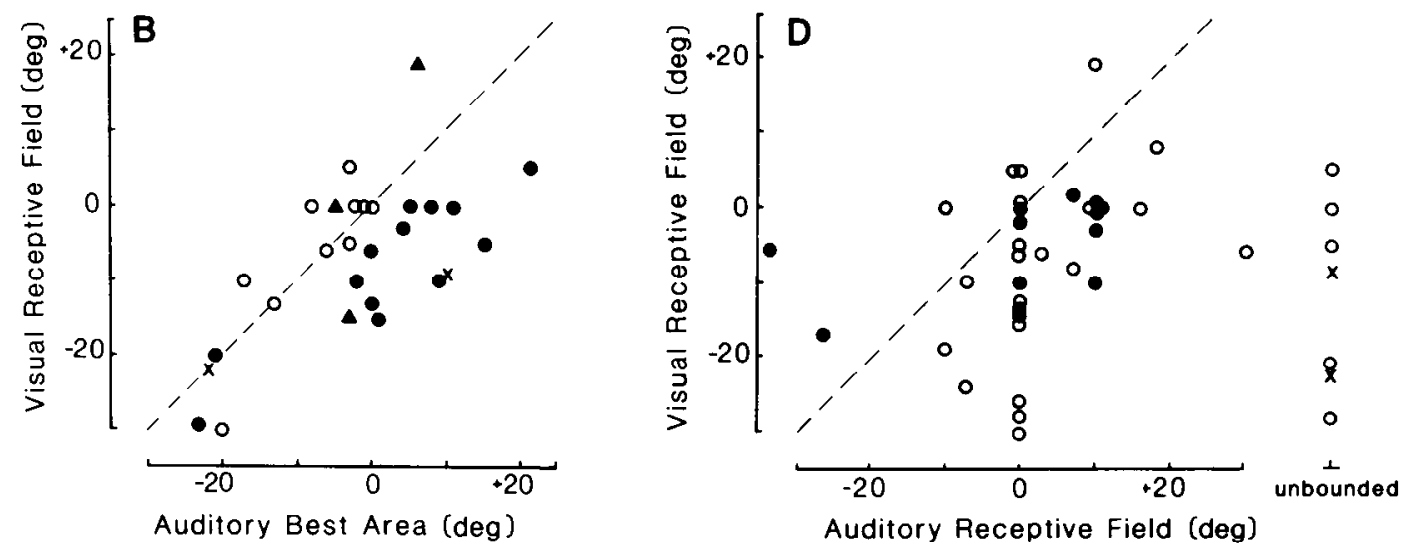

\section{ELEVATION}

Figure 11. Relationship of auditory best areas and receptive ficlds to visual receptive ficlds. Visual data are from auditory-visual bimodal units or, in the case of unimodal auditory units, from nearby visual units. The locations of auditory best areas $(A$ and $B)$ and receptive fields $(C$ and $D)$ are given by their geometrical centers. Hemifield, frontal, and omnidirectional units are represented by open circles, solid circles, and $X$, respectively. Solid triangles in $A$ represent the best areas of units for which auditory receptive field borders were not measured. Dashed lines indicate perfect auditory and visual correspondence. The receptive fields of omnidirectional units are unbounded in azimuth and elevation and those of many hemifield units are unbounded in elevation. Thus, in $C$ and $D$, the location on the $a b s c i s s a$ of points representing these units is arbitrary. No correction was made for eye position, except that visual data were discarded when the eyes appeared to be moving or the optic disks were more than $5^{\circ}$ from their resting positions.

muth profiles of three units recorded along a rostral-to-caudal line of electrode penetrations in one cat. The best areas shifted in azimuth from near the frontal midline for the most rostral unit to contralateral $87^{\circ}$ for the most caudal unit. All of these best areas were centered near $0^{\circ}$ elevation. Best areas varied in elevation as a function of mediolateral unit position. For example, Figure $12 B$ illustrates the elevation tuning of three units recorded along a mediolateral line in one cat. The centers of best areas shifted progressively lower from $+9^{\circ}$ for the most medial unit to $-21^{\circ}$ for the most lateral unit. These best areas all were centered in azimuth between contralateral 20 and $34^{\circ}$.

Contour plots show the orientation in the superior colliculus of the maps of auditory azimuth and elevation (Fig. 13). The axis of changing azimuth is oriented rostrocaudally and contours of constant azimuth are oriented approximately mediolaterally. The axis of changing elevation is oriented mediolaterally, and contours of constant elevation are approximately parallel to the lateral margin of the superior colliculus.

The representation of sound elevation in the mediolateral dimension of the superior colliculus was complicated somewhat by the fact that some units recorded at the medial edge of the intermediate gray layer showed unusually broad spatial tuning. The locations of the centers of these large best areas often conformed to the topography predicted by nearby recordings, but in several cases we encountered abrupt shifts in the elevation and azimuth of best areas when mapping near the medial edge of the superior colliculus.

Receptive fields also varied in location as a function of unit position, but this variation was less orderly than the progression of best area locations. The locations in azimuth of best areas varied continuously and nearly linearly with the rostrocaudal position of units (Fig. 14A). In contrast, the range of receptive field locations contained a substantial discontinuity (Fig. 14B). The leading edges of receptive fields varied somewhat with the rostrocaudal positions of units, but leading edges covered a smaller range of azimuth than did best areas, and units with leading edges located at the frontal midline were encountered throughout most of the rostrocaudal extent of the intermediate gray layer (Fig. $14 \mathrm{C}$ ). In the mediolateral dimension, the elevation of the receptive fields of frontal units showed a dependence on unit position similar to that of best areas (Fig. 15). However, omnidirectional units and many hemifield units lacked receptive field borders in elevation even though their best areas were well defined in elevation.

All units recorded along a given electrode penetration tended to have similar best areas, regardless of the layer in which they 
were recorded. Thus, the maps of auditory space in the intermediate white and deep gray layers were essentially parallel to that in the intermediate gray layer. However, we could not combine mapping data from different layers without degrading the precision of the map in any one layer. This was due in part to the oblique orientation of the electrode penetrations. Other factors are that the deep gray layer is of nonuniform thickness and is not coextensive with the more superficial layers, and that some of our recordings in the intermediate white layer could have been from fibers of passage.

The spatial pattern of activity in the superior colliculus. We used the spatial response profiles of single units distributed throughout the intermediate gray layer to compute the spatial pattern of activity elicited by a sound at a given location. Patterns in the rostrocaudal and mediolateral dimensions are shown in Figures 16 and 17, respectively. The length of each bar indicates the response of one unit relative to its maximum response, and the open symbols indicate inactive units. The active units are those having receptive fields which contain the specified sound source location. The caudal and rostral borders between active and inactive cells correspond to, respectively, the leading and trailing (most peripheral) edges of receptive fields. The best areas of units are manifested in the spatial pattern of activity as a restricted region of units responding within $75 \%$ of their maximum firing rate.

Changes in the azimuth of a sound source resulted in changes in the rostrocaudal distribution of active units. Frontal sounds activated rostral units, and peripheral sounds activated units located further caudal. However, a sound located anywhere on the contralateral side of the cat activated units over a large portion of the superior colliculus. For example, sounds located between contralateral 20 and $40^{\circ}$ were within the receptive fields of most units distributed throughout the entire rostrocaudal extent of the intermediate gray layer. Consequently, changes in the location of a sound source within that range resulted in little or no change in the overall distribution of active units. Moreover, sounds located beyond about contralateral $70^{\circ}$ were outside of the receptive fields of all frontal units yet within the fields of all hemifield units, so that there was virtually no change in the distribution of active units corre-

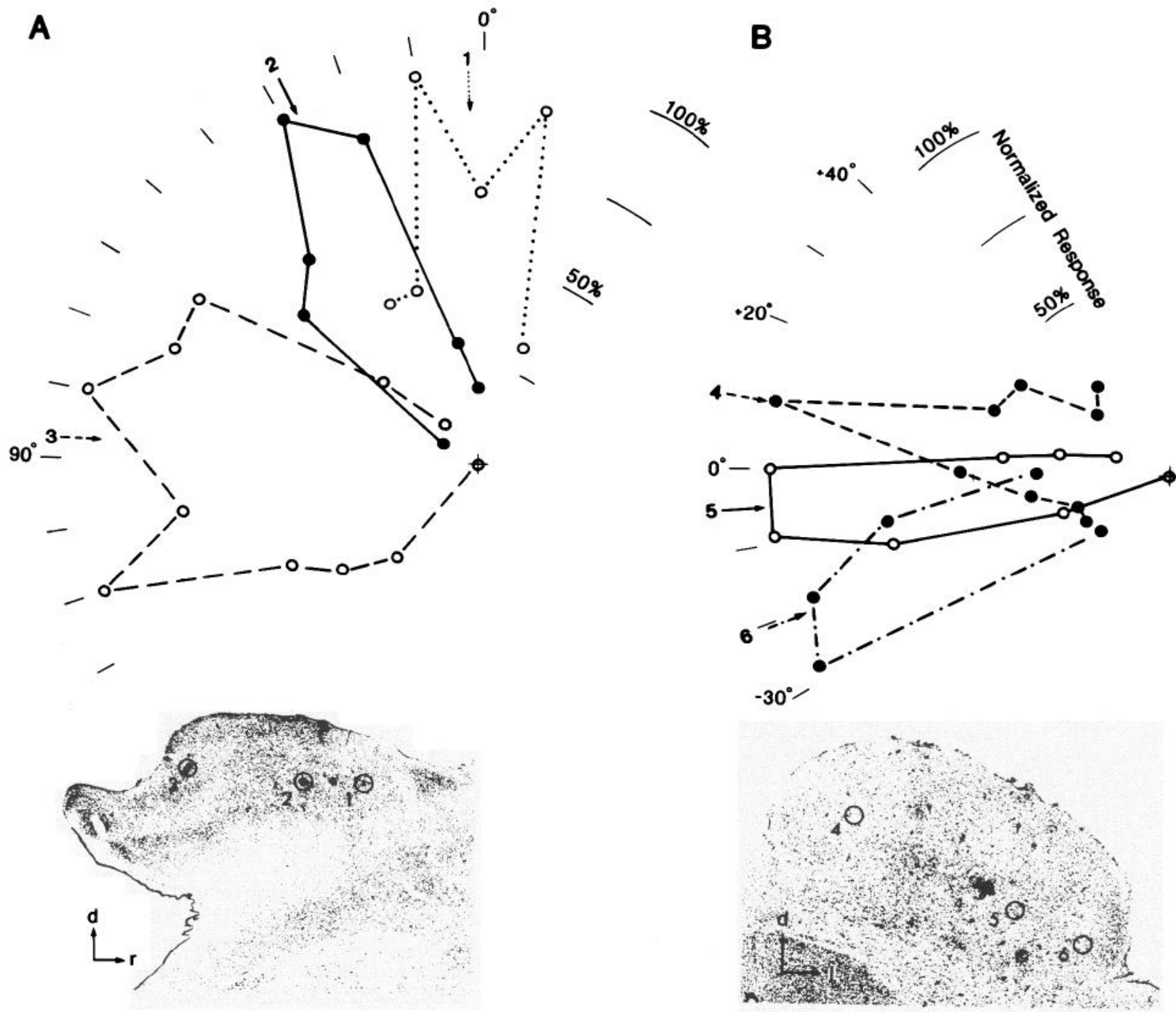

Figure 12. Spatial tuning associated with different unit positions in the superior colliculus. Spatial response profiles are shown for six units located along a rostrocaudal $(A)$ and a mediolateral $(B)$ series of electrode penetrations through the intermediate gray. Profiles in azimuth $(A)$ and elevation $(B)$ are shown, and the positions of the corresponding units are shown in the accompanying parasagittal $(A)$ and transverse $(B)$ sections. The elevation profiles in $B$ were measured at contralateral $20^{\circ}$ azimuth. Unit firing rates are normalized according to the maximum firing rate of each unit. The maximum mean response rates for units 1 to 6 were 2, 2.6, 0.8, 2.1, 0.9 , and 1.1 spikes per presentation, respectively. Numbered arrows indicate the centers of best areas, and numbered circles indicate the sites of marking lesions present in these or nearby sections. All units were in the intermediate gray layer. $d$, dorsal; $r$, rostral; $l$, lateral. Scale $=0.5 \mathrm{~mm}$. 


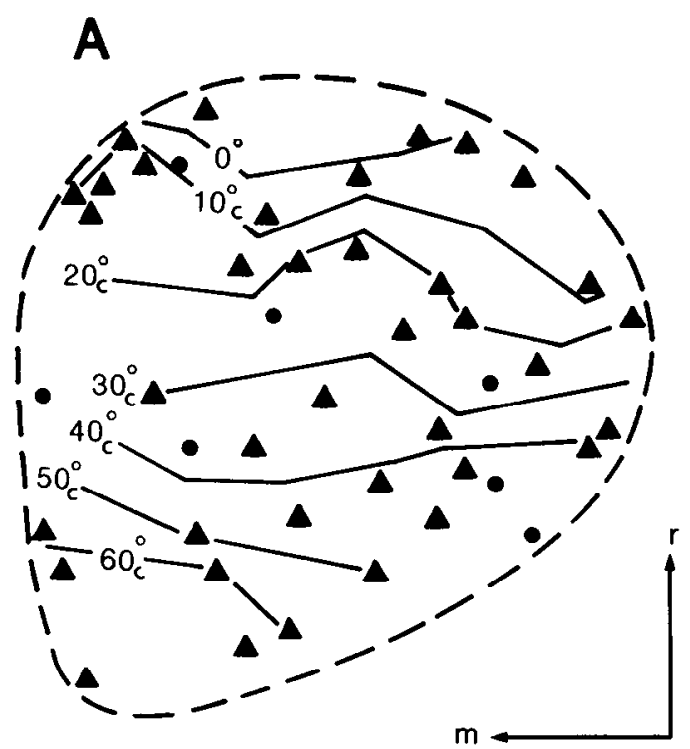

AZIMUTH

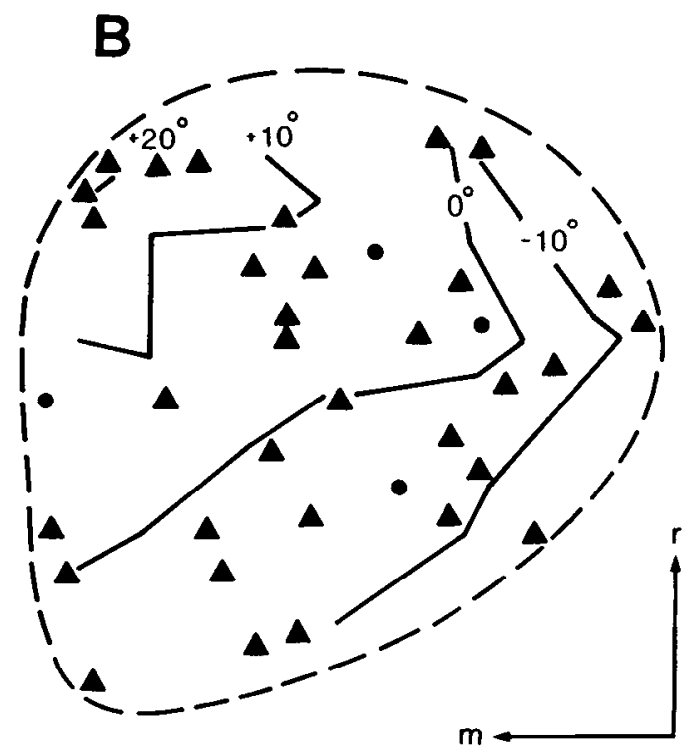

ELEVATION

Figure 13. Maps of auditory azimuth and elevation in the intermediate gray layer. The intermediate gray layer is shown in dorsal views. The contours (solid lines) were drawn strictly according to the best areas of units recorded at sites indicated by solid triangles. Best areas measured at some sites (indicated by dots) differed by 10 to $20^{\circ}$ from the values predicted by the other data. These data were disregarded when drawing the contours. $c$, contralateral; $r$, rostral; $m$, medial. $S c a l e=1 \mathrm{~mm}$.

A

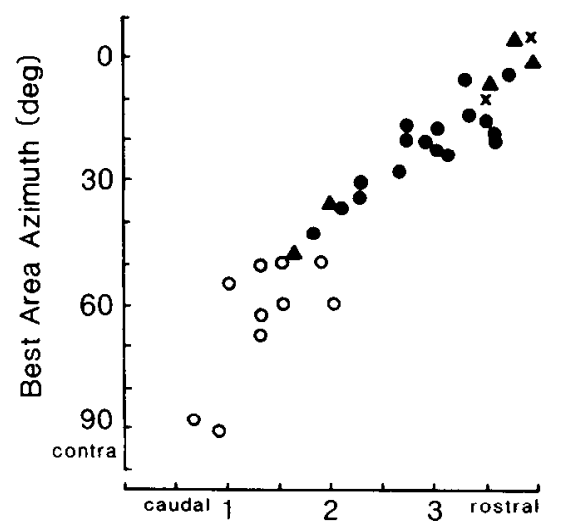

B

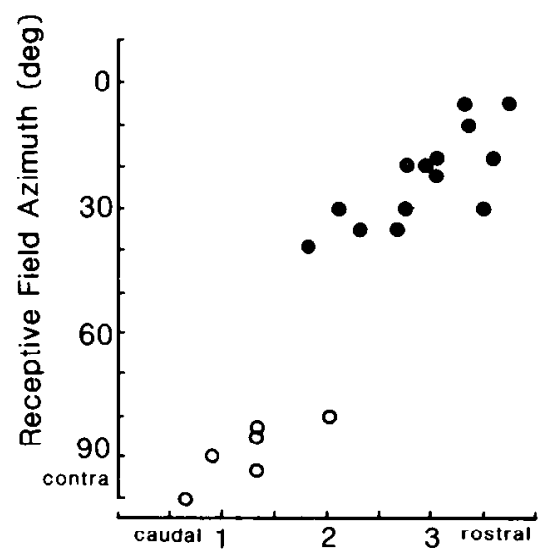

Superior Colliculus Location (mm)

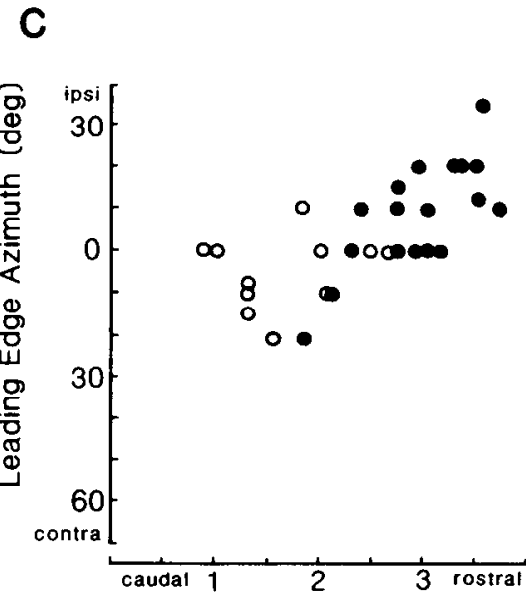

Figure 14. Three features of azimuth tuning related to rostrocaudal unit position. The locations in azimuth of best areas $(A)$, receptive field centers $(B)$, and receptive field leading edges $(C)$ are plotted according to the positions of units in the rostrocaudal dimension of the intermediate gray layer. Rostrocaudal position was measured from the most caudal histological section passing through the superficial layers. Open and sulid circles and $X$ represent hemifield, frontal, and omnidirectional units, respectively. In $A$, solid triangles indicate the best areas of units for which receptive field borders were not measured. Omnidirectional units are not represented in $B$ and $C$.

sponding to shifts in the location of a sound between approximately 70 and $140^{\circ}$ contralateral. Finally, there was no sound location for which the region of active cells was bounded both rostrally and caudally by regions of inactive cells.

In contrast, the focus of greatest activity varied continuously in location with changes in the location of a sound source within the frontal contralateral quadrant. The location of the region of maximal activity mapped even the locations between contralateral 20 and $40^{\circ}$ for which there is no change in the overall distribution of active units. Although few best areas extended behind the cat's head, sound locations within the posterior contralateral quadrant nevertheless were signaled by the relative activity of units located caudally.

The elevation of a sound determined the spatial pattern of activity in the mediolateral dimension of the superior colliculus (Fig. 17). The pattern was influenced strongly by the units located near the medial edge of the layer that displayed unusually broad elevation tuning. Sounds located higher than about $+30^{\circ}$ activated only medial units. However, sound sources located within $10^{\circ}$ of the horizontal plane activated nearly all of the units in this sample. Units activated by a sound source at $-30^{\circ}$ occupied two discrete regions: one located laterally and 

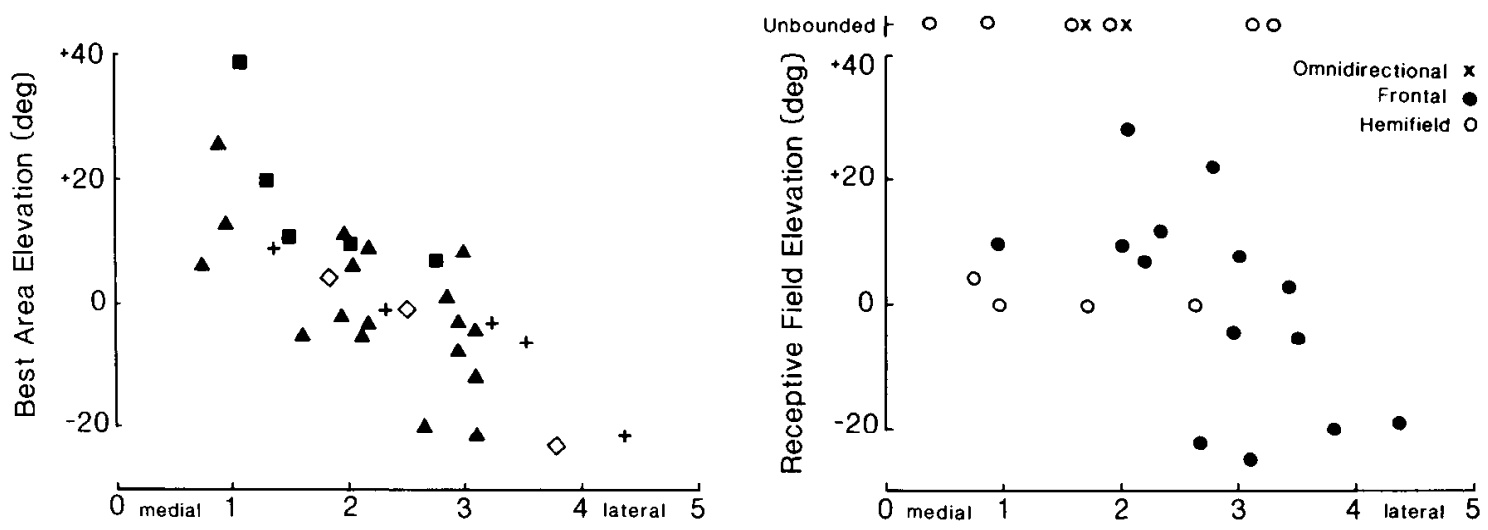

Superior Colliculus Location (mm)

Figure 15. 'I'wo features of elevation tuning related to mediolateral unit position. The locations in elevation of the geometric centers of best areas $(A)$ and receptive fields $(B)$ are plotted against the positions of units in the mediolateral dimension of the intermediate gray layer. Mediolateral position was measured relative to the midsagittal plane. In $A$, the solid squares, open diamonds, and plus signs represent units recorded in each of three mediolateral series of penetrations in three cats. The solid triangles represent units recorded in additional penetrations in additional cats. In $B$, open and solid circles and $X$ represent hemifield, frontal, and omnidirectional units. The receptive fields of omnidirectional and many hemifield units were unbounded in elevation; the location on the ordinate of symbols representing these units is arbitrary.

a smaller one located medially containing the broadly tuned units. The focus of maximally activated units shifted continuously from medial to lateral in relation to progressively lower sound locations, although a few units located medially responded strongly to all but the lowest sound locations.

\section{Discussion}

Units in the cat's superior colliculus are selective for the location of a sound source, and this selectivity shifts systematically as a function of unit position. The orderly topography of spatial tuning results in a distribution of activity which maps the locations of sounds. The data reveal the relative contributions of various features of spatial tuning to this map of auditory space.

Spatial tuning of single units. The spatial response profile of a unit in the superior colliculus shows an optimal stimulus location within a broader receptive field. This observation is consistent with previous descriptions of units in the owl's midbrain (Knudsen and Konishi, 1978; Knudsen, 1982) and the guinea pig's superior colliculus (King and Palmer, 1983). The receptive field of a unit provides a measure of the maximum area to which a given unit is sensitive. The optimal stimulus location of a unit was approximated by its best area, the region within which sounds elicited a response within $75 \%$ of the unit's maximum firing rate. A criterion higher than the $75 \%$ level might have specified the optimal stimulus location more closely, but best area measurements would have been more vulnerable to irregularities in response profiles.

Units form three classes on the basis of properties of their receptive fields. The receptive fields of hemifield units extend behind the cat's head, while those of frontal units are contained entirely in front of the interaural transverse plane. Omnidirectional units have unbounded receptive fields and form a relatively small part of our sample. Omnidirectional units resemble frontal units with regard to their position in the superior colliculus and to the locations of their auditory best areas and visual receptive fields. This suggests that omnidirectional units may be regarded as frontal units that are insensitive to the auditory cues that establish sharp receptive field borders.

The receptive fields of frontal units resemble those of the "axial" units that are found in the cat's primary auditory cortex (Middlebrooks and Pettigrew, 1981). Both frontal and axial fields are contained entirely in front of the cat's head. In addition, the fields of some of the axial units, like those of most of the frontal units, are insensitive to changes in stimulus intensity. However, the fields of axial units all are centered on the axis of greatest sensitivity of the contralateral external ear, whereas the geometrical centers of the fields of frontal units are distributed across a range of about $40^{\circ}$ of azimuth.

Auditory receptive fields in the cat's superior colliculus have been described by several other groups. Gordon (1973) mapped the receptive fields of units in the intermediate and deep layers using hand-held auditory stimuli. She found that auditory units had receptive field borders, but frequently only the most frontal border, or "leading edge," could be determined with confidence. However, she presented auditory stimuli against a tangent screen located in front of the cat; thus it would have been impossible to measure the most peripheral edges of most fields, particularly those of the hemifield units. In the data presented by Harris et al. (1980), the centers of receptive fields were distributed continuously throughout the range of $0^{\circ}$ to contralateral $65^{\circ}$. This observation conflicts with our findings that no receptive fields are centered between contralateral 40 and $70^{\circ}$ and that about half of the fields are centered peripheral to contralateral $70^{\circ}$. Again, sound locations behind the cat's head were not tested by the Harris group, so that the apparent receptive field centers of our hemifield units might have been displaced frontally in that study. These groups found that the leading edges (Gordon, 1973) or the centers (Harris et al., 1980) of auditory and visual receptive fields tended to correspond in azimuth. In contrast, we found correspondence of auditory and visual receptive fields only among the frontal units, although there was a general correspondence between the visual receptive fields and auditory best areas of all units.

The discrepancies between our results and those in the earlier reports probably are due to differences in the response criteria used to measure auditory receptive field borders. We considered any stimulus that excited a unit to be within its receptive field. For many units, the receptive field defined in this way extended well beyond the steepest point in the spatial response profile. Although the earlier reports did not specify the criteria used to 
define receptive fields, it seems likely that the stated receptive field borders correspond to the locations at which the responses of units fell off sharply. Thus, the "receptive fields" measured in the earlier studies probably correspond more closely to the "best areas" that we have described. An additional factor in the study by the Harris group is that the cats were awake and the external ears were free to move. In a preliminary report, Wise et al. (1982) described results obtained using methods more comparable to ours. They found that $60 \%$ of units had receptive fields that filled the contralateral hemifield and the remainder had fields that were restricted within the frontal contralateral quadrant. This is more consistent with our observations.

The spatial tuning of units in the superior colliculus is relatively insensitive to changes in stimulus intensity. Near a unit's threshold, response probabilities are low and spatial profiles are difficult to measure reliably. However, for stimuli more than about $10 \mathrm{~dB}$ above threshold, a unit's response is dictated much more by the location of the stimulus than by its intensity. The thresholds of superior colliculus units are at least. $10 \mathrm{~dB}$ higher than those of most units in the primary auditory pathway (e.g., in the inferior colliculus; unpublished observa-
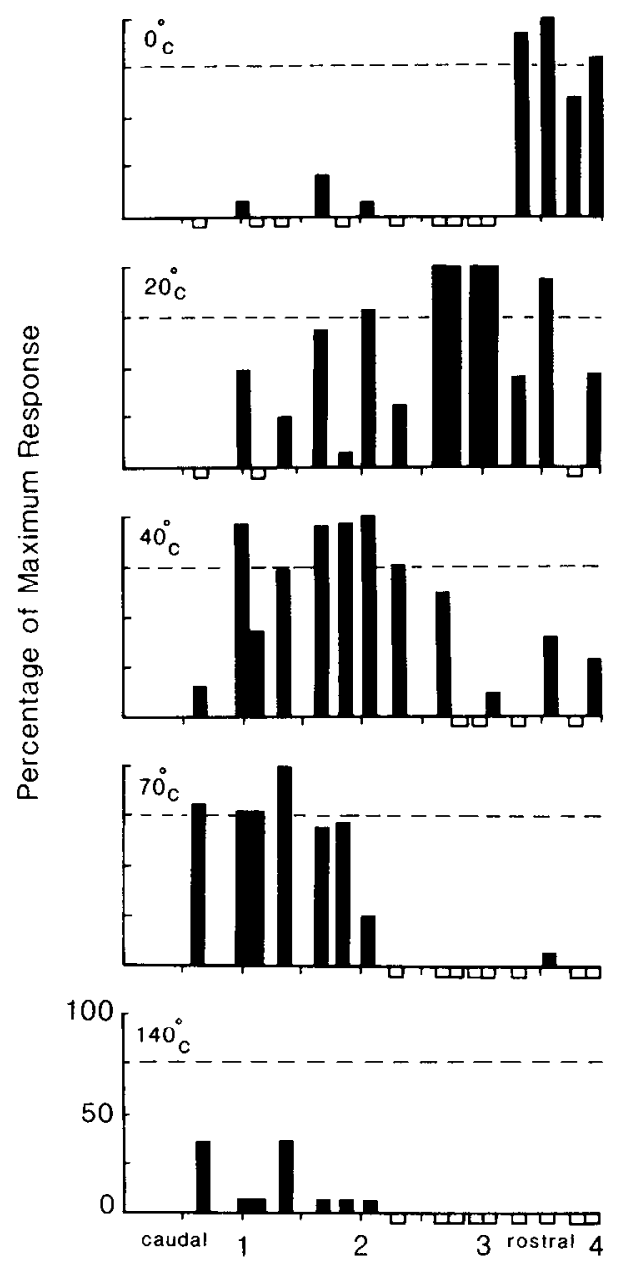

Superior Colliculus Location ( $\mathrm{mm}$ )

Figure 16. The spatial pattern of unit activity in the rostrocaudal dimension of the intermediate gray layer. The patterns resulting from sound sources at five different locations in the horizontal plane are represented by the activity of 16 units selected for best areas centered in elevation hetween +10 and $-10^{\circ}$. The lengths of bars indicate the activity of units relative to their maximum responses, and the open symbols indicate inactive units (i.e., units for which the specified sound source is out of the unit's receptive field). The dashed lines indicate the $75 \%$ criterion. $c$, contralateral.

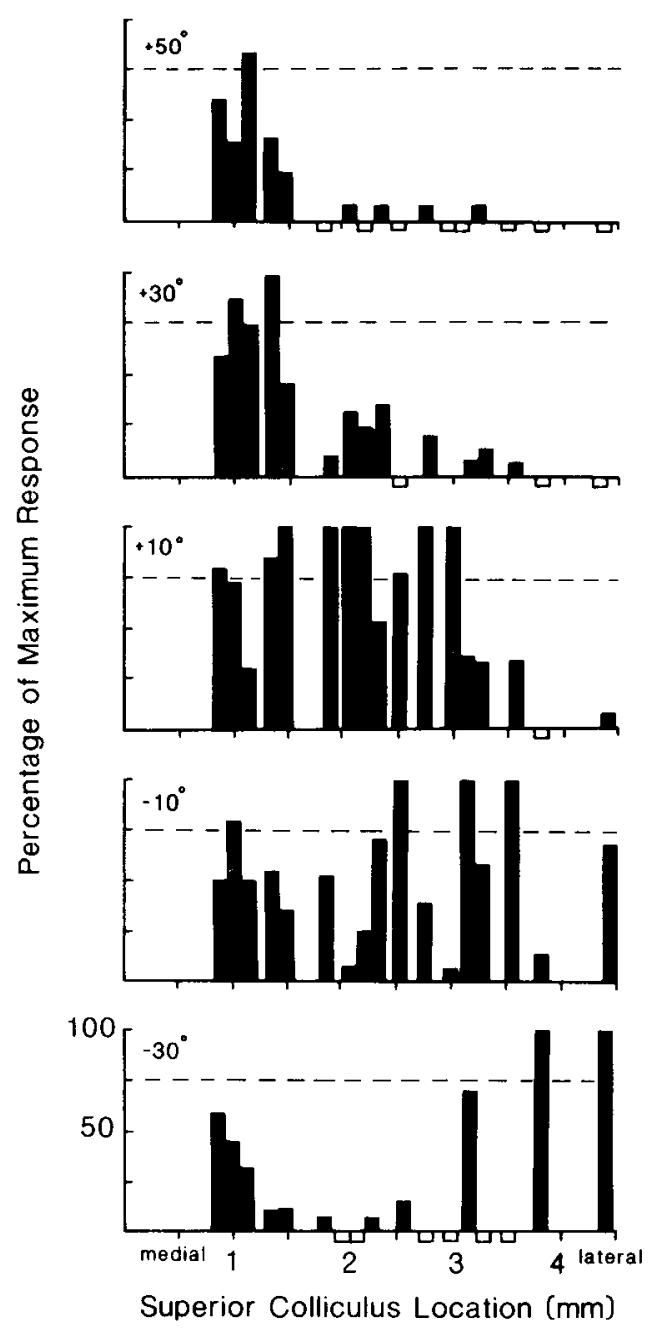

Figure 17. The spatial pattern of unit activity in the mediolateral dimension of the intermediate gray layer. The patterns resulting from sound sources at five different locations in elevation are represented by the activity of 17 units selected for best areas centered in azimuth between contralateral 5 and $35^{\circ}$. See Figure 16 for further details.

tions). Thus, sounds $10 \mathrm{~dB}$ greater than the threshold of a superior colliculus unit would be at least $20 \mathrm{~dB}$ above the threshold of many units in the primary pathway and would be strong enough to activate inputs arising at either ear regardless of the location of the sound. This suggests that the spatial response profiles derived using intensities as low as $10 \mathrm{~dB}$ above threshold could show the effects of binaural interactions. Moreover, it raises the possibility that at least some superior colliculus units require binaural stimulation for a strong response. Binaural interactions would enable the auditory system to utilize binaural cues for sound location that are independent of sound source intensity.

The intensity invariance of superior colliculus units contrasts with the properties of some units described in other auditory structures in the cat. In the inferior colliculus, auditory receptive fields of units expand dramatically with increasing intensity (Semple et al., 1983). For example, several illustrated units that showed hemifield receptive fields at one intensity responded to sounds at all locations when the intensity was increased by $20 \mathrm{~dB}$. Similarly, the receptive fields of many units in the cat's auditory cortex change with changes in stimulus intensity, although the receptive fields of others are quite resistant to changes in stimulus intensity (Middlebrooks and Pettigrew, 1981). 
Acoustical cues for spatial selectivity. The location of a sound source is signaled by interaural delay cues and by intensity cues. Interaural delays result from differences in the lengths of the paths of sound to each ear. Interaural delays are nearly equal for all sounds located at a constant azimuth (on a "cone of confusion;" Woodworth, 1938). Sounds located at different azimuths produce different interaural delays. Thus, interaural delays could provide cues to auditory azimuth but not elevation.

Intensity effects provide both monaural and binaural cues to the location of a sound. Depending on the location of the source, the intensity of a sound is increased or decreased by the collecting and shadowing properties of the head and external ears (Wiener et al., 1966; Middlebrooks and Pettigrew, 1981; Phillips et al., 1982). These effects increase with increasing sound frequency. Thus, the frequency spectrum of a sound is shaped differently depending on the location of the source in azimuth and elevation. In humans, properties of the spectra of sounds arriving at each ear drum provide the essential cues for vertical sound localization (Gardner and Gardner, 1973; Hebrank and Wright, 1974; Morimoto and Ando, 1980). In the cat's superior colliculus, monaural spectra likewise could provide cues for elevation tuning of units and might also contribute to azimuth tuning.

Interaural differences in spectra are regarded as important cues to the azimuth of a sound; interaural spectral differences are equivalent to interaural intensity differences measured at multiple frequencies. Yet, for sounds located off of the median plane, these interaural differences also vary with elevation and, thus, could contribute to elevation selectivity. The broad frequency tuning of units in the superior colliculus and their sensitivity to broad band sounds are consistent with the possibility that monaural and/or binaural spectral cues contribute to their spatial selectivity.

Wise and Irvine (Wise and Irvine, 1983; Irvine and Wise, 1983) measured the selectivity of units in the cat's superior colliculus for interaural intensity differences. Units located rostrally responded best when the intensities at the two ears were nearly equal. Caudal units responded best to monaural stimulation of the contralateral ear and decreased their responses as the intensity of the ipsilateral stimulus was increased. The interaural intensity difference at which the responses of the caudal units cut off most sharply varied with rostrocaudal unit position. Wise and Irvine (Irvine and Wise, 1983; Wise and Irvine, 1983) predicted that these units would show hemifield receptive fields with leading edges that would vary in azimuth according to unit position. Our results confirm that caudal units have hemifield receptive fields, yet the locations of the leading edges of these fields show no systematic relationship to unit position (Fig. $14 \mathrm{C}$, open symbols). The variation in interaural intensity selectivity with rostrocaudal unit position reported by Wise and Irvine (Wise and Irvine, 1983 ; Irvine and Wise, 1983) is consistent with the variation in best area azimuths that we observe.

The sharpness of spatial selectivity varies markedly among different units, even among units with similar best area locations. For example, best areas centered in azimuth around contralateral $50^{\circ}$ vary in width between approximately 15 and $70^{\circ}$ (Fig. $8 A$ ). This variation is substantially greater than any observed change in tuning resulting from a change in stimulus intensity (e.g., Fig. 10). There is no apparent correlation of sharp or broad tuning with particular cell layers, no strict relationship between sharpness of tuning and the locations of best areas, and no relationship between the sharpness of auditory tuning and the size of visual receptive fields. One interpretation is that the sharply and broadly tuned units respond to different sets of spatial cues that nevertheless correspond to the same location in space. The sensitivity to different cues might ensure that at least some subset of units would be capable of representing the location of any sound regardless of its spectral composition.

One small population of units was characterized by particularly broad spatial tuning. These units were located at the medial edge of the superior colliculus and had best areas that were greater than $40^{\circ}$ in vertical extent. Most of these large best areas were centered above the horizontal plane. The sites where these units were recorded could not be distinguished by histological criteria from the sites where more sharply tuned units were recorded. The comparative lack of selectivity of the medial units suggests that their contribution to the function of the superior colliculus is fundamentally different from that of more spatially selective units. However, we also note that only these broadly tuned units respond strongly to sounds located higher than approximately $+20^{\circ}$. Perhaps the role of these units is simply to provide a representation of high elevations.

Our data were collected from a preparation in which the ears were fixed in place, yet a behaving cat can make independent movements of each ear. Movements of the ear shift the axis of greatest sensitivity, especially for sound frequencies greater than about $10 \mathrm{kHz}$ (Phillips et al., 1982). Units in the superior colliculus respond to high frequency sounds; thus, one would expect that movements of the ears would result in marked changes in spatial tuning unless the superior colliculus can use information regarding the positions of the ears to compensate for alterations in their acoustical properties. The effects of changes in ear position on the spatial tuning of superior colliculus units or on the map of auditory space remain to be determined.

A neural code for auditory space. We can characterize the representation of auditory space by inferring the spatial pattern of activity elicited in the superior colliculus by a sound at a given location. Because of the intensity invariance of the spatial tuning of most units, the pattern is largely insensitive to changes in stimulus intensity. This analysis is somewhat analogous to the computation of the superior colliculus "image" of a point in visual space (McIlwain, 1975). A sound elicits activity in a large population of units that, for some sound locations, can include nearly all of the auditory units in the superior colliculus. Activity is graded topographically within this population so that the units activated to near their maximum firing levels are clustered to form a single focus of greatest activity. Since maximum firing rates vary substantially among different units, the definition of a response criterion relative to a unit's maximum firing rate assumes that a neuron that receives its input from the superior colliculus can regulate its sensitivity to accommodate the normal range of firing rates of each input.

It is incorrect to regard the spatial pattern of activity in the superior colliculus as a restricted region of active units bounded on all sides by inactive units. As a sound source is positioned successively further contralaterally, the region of active units does shift caudally. However, the activated region is never bordered both rostrally and caudally by inactive units, and for sound sources located between contralateral 20 and $40^{\circ}$, it fills the entire rostrocaudal extent of the superior colliculus. In contrast, the area of units activated to near their maximum firing rates is bordered both rostrally and caudally by units firing at a lower rate for many sound locations. The position of this focus of greatest activity maps sound locations throughout the frontal contralateral quadrant.

The representation of a visual stimulus in the intermediate gray layer appears to share several properties with the auditory representation. The visual receptive fields of units in the intermediate gray layer are substanitally larger than those of units in more superficial layers. For example, Gordon (1973) reported that approximately $60 \%$ of units recorded in the layers deep to the optic layer had receptive fields that were wider than $20^{\circ}$, and some units had fields which occupied the entire half of the 
tangent screen contralateral to the recording site. These visual fields are comparable in size to the auditory receptive fields that we have described. Thus, we can infer that the units in the intermediate gray layer activated by stimuli in contralateral visual space occupy large fractions of the area of the layer, probably greater than one-half. The observation that units in the deeper layers display optimal stimulus locations within their visual receptive fields (Goldberg and Wurtz, 1972), as within their auditory fields, suggests that visual space might also be coded in the intermediate gray layer in terms of foci of maximal activity.

Conclusion. We have explored the spatial tuning of units in the superior colliculus in order to identify features that could constitute a map of auditory space. The receptive field of a unit indicates the total area within which a sound will excite the unit. Discrete classes of units are distinguished by characteristics of their receptive fields, and the segregation of units belonging to different receptive field classes further supports this classification. However, the geometric centers and the leading edges of receptive fields map only limited or discontinuous regions of auditory space. Spatial topography is most evident when the responses of units are evaluated quantitatively relative to their maximum firing rates. The optimal stimulus locations of units, their best areas, vary continuously in azimuth and elevation as a function of unit position throughout the superior colliculus, and best areas correspond most consistently with visual receptive fields. Within the spatial pattern of activity in the superior colliculus, the location of a sound appears to be coded by the location of the focus of greatest activity within a broad area of activated units.

\section{References}

Bishop, P. O., W. Kozak, and G. J. Vakkur (1962) Some quantitative aspects of the cat's eye: Axis and plane of reference, visual field coordinates, and optics. J. Physiol. (Lond.) 163: 466-502.

Chalupa, L. M., and R. W. Rhoades (1977) Response of visual, somatosensory, and auditory neurones in the golden hamster's superior colliculus. J. Physiol. (Lond.) 270: 595-626.

Dräger, U. C., and D. H. Hubel (1975) Responses to visual stimulation and relationship between visual, auditory, and somatosensory inputs in mouse superior colliculus. J. Neurophysiol. 38: 690-713.

Dräger, U. C., and D. H. Hubel (1976) Topography of visual and somatosensory projections to mouse superior colliculus. J. Neurophysiol. 39: 91-101.

Gardner, M. B., and R. S. Gardner (1973) Problem of localization in the median plane: Effect of pinnae cavity occlusion. J. Acoust. Soc. Am. 53: 400-408.

Goldberg, M. E., and R. H. Wurtz (1972) Activity of superior colliculus cells in behaving monkey. I. Visual receptive fields of single neurons. J. Neurophysiol. 35: 542-559.

Gordon, B. (1973) Receptive fields in deep layers of cat superior colliculus. J. Neurophysiol. 36: 157-178.

Green, C. J., J. Knight, S. Precious, and S. Simpkin (1981) Ketamine alone and combined with diazepam or xylazine in laboratory animals: A 10 year experience. Lab. Anim. 15: 163-170.

Harris, L. R., C. Blakemore, and M. Donaghy (1980) Integration of visual and auditory space in the mammalian superior colliculus. Nature 288: $56-59$
Hebrank, J., and D. Wright (1974) Spectral cues used in the localization of sound sources in the medial plane. J. Acoust. Soc. Am. 56: 18291834.

Irvine, D. R. F., and I. Z. Wise (1983) Topographic organization of interaural-intensity-difference sensitivity and the representation of auditory azimuthal location in the deep layers of the superior colliculus. In Mechanisms of Hearing, W. R. Webster and L. M. Aitkin, eds., pp. 101-106, Monash University Press, Clayton, Australia.

Kanaseki, T., and J. M. Sprague (1974) Anatomical organization of pretectal nuclei and tectal laminae in the cat. J. Comp. Neurol. 158: 319-337.

King, A. J., and A. R. Palmer (1983) Cells responsive to free-field auditory stimuli in guinea-pig superior colliculus: Distribution and response properties. J. Physiol. (Lond.) 342: 361-381.

Knudsen, E. I. (1982) Auditory and visual maps of space in the optic tectum of the owl. J. Neurosci. 2: 1177-1194.

Knudsen, E. I., and M. Konishi (1978) Space and frequency are represented separately in auditory midbrain of the owl. J. Neurophysiol. $41: 870-884$.

McIlwain, J. T. (1975) Visual receptive fields and their images in superior colliculus of the cat. J. Neurophysiol. 38: 219-230.

Middlebrooks, J. C., and J. D. Pettigrew (1981) Functional classes of neurons in primary auditory cortex of the cat distinguished by sensitivity to sound location. J. Neurosci. 1: 107-120.

Morgan, L. O. (1926) Myelin hematoxylin stain. Anat. Rec. 32: 283.

Morimoto, M., and Y. Ando (1980) On the simulation of sound localization. J. Acoust. Soc. Jpn. (E) $1:$ 167-174.

Phillips, D. P., M. B. Calford, J. D. Pettigrew, L. M. Aitkin, and M. N. Semple (1982) Directionality of sound pressure transformation at the cat's pinna. Hear. Res. 8: 13-28.

Roucoux, A., M. Crommelinck, and D. Guitton (1981) The role of the superior colliculus in the generation of gaze shift. In Progress in Oculomotor Research, A. F. Fuchs and W. Becker, eds., pp. 129-135, Elsevier-North Holland Publishing Co., New York.

Semple, M. N., L. M. Aitkin, M. B. Calford, J. D. Pettigrew, and D. P. Phillips (1983) Spatial receptive fields in the cat's inferior colliculus. Hear. Res. 10: 203-215.

Sprague, J. M., and T. H. Meikle, Jr. (1965) The role of the superior colliculus in visually guided behavior. Exp. Neurol. 11: 115-146.

Stein, B. E., and H. P. Clamann (1981) Control of pinna movements and sensorimotor register in cat superior colliculus. Brain Behav. Evol. 19: 180-192.

Stein, B. E., B. Magalhaew-Castro, and L. Kruger (1976) Relationship between visual and tactile representations in cat superior colliculus. J. Neurophysiol. 39: 401-419.

Sterling, P., and B. G. Wickelgren (1969) Visual receptive fields in the superior colliculus of the cat. J. Neurophysiol. 32: 1-15.

Tiao, Y-C., and C. Blakemore (1976) Functional organization in the superior colliculus of the golden hamster. J. Comp. Neurol. 168: 483504.

Wiener, F. M., R. R. Pfeiffer, and A. S. N. Backus (1966) On the sound pressure transformation by the head and auditory meatus of the cat. Acta Otolaryngol. 61: 255-269.

Wise, L. Z., and D. R. F. Irvine (1983) Auditory response properties of neurons in deep layers of cat superior colliculus. J. Neurophysiol. 49: $674-685$.

Wise, L. Z., D. R. F. Irvine, J. D. Pettigrew, and M. B. Calford (1982) Auditory spatial receptive field properties of neurons in intermediate deep layers of cat superior colliculus. Neurosci. Lett. Suppl. 8: S88.

Woodworth, R. S. (1938) Experimental Psychology, Holt, Rinehart, and Winston. New York.

Wurtz, R. H., and J. E. Albano (1981) Visual motor function of the primate superior colliculus. Annu. Rev. Neurosci. 3: 189-226. 